\title{
Ferromagnetic Resonance
}

\author{
Orhan Yalçın
}

Additional information is available at the end of the chapter

http://dx.doi.org/10.5772/56134

\section{Introduction}

Ferromagnetism is used to characterize magnetic behavior of a material, such as the strong attraction to a permanent magnet. The origin of this strong magnetism is the presence of a spontaneous magnetization which is produced by a parallel alignment of spins. Instead of a parallel alignment of all the spins, there can be an anti-parallel alignment of unequal spins. This results in a spontaneous magnetization which is called ferrimagnetism.

The resonance arises when the energy levels of a quantized system of electronic or nuclear moments are Zeeman split by a uniform magnetic field and the system absorbs energy from an oscillating magnetic field at sharply defined frequencies corresponding to the transitions between the levels. Classically, the resonance event occurs when a transverse ac field is applied at the Larmor frequency.

The resonance behaviour usually called magnetic resonance (MR) and nuclear magnetic resonance (NMR). Main types of resonance phenomenon can be listed as nuclear magnetic resonance (NMR), nuclear quadrupole resonance (NQR), electron paramagnetic/spin resonance (EPR, ESR), spin wave resonance (SWR), ferromagnetic resonance (FMR), antiferromagnetic resonance (AFMR) and conductor electron spin resonance (CESR). The resonant may be an isolated ionic spin as in electron paramagnetic resonance (EPR) or a nuclear magnetic resonance (NMR). Also, resonance effects are associated with the spin waves and the domain walls. The resonance methods are important for investigating the structure and magnetic properties of solids and other materials. These methods are used for imaging and other applications.

The following information can be accessed with the help of such resonance experiments. (i) Electrical structure of point defects by looking at the absorption in a thin structure. (ii) The line width with the movement of spin or surroundings isn't changed. (iii) The distribution of the magnetic field in solid by looking at the of the resonance line position (chemical shift and etc.). (iv) Collective spin excitations. 
The atoms of ferromagnetic coupling originate from the spins of $d$-electrons. The size of $\mu$ permanent atomic dipoles create spontaneously magnetized. According to the shape of dipoles materials can be ferromagnetic, antiferromagnetic, diamagnetic, paramagnetic and etc.

Ferromagnetic resonance (FMR) technique was initially applied to ferromagnetic materials, all magnetic materials and unpaired electron systems. Basically, it is analogous to the electron paramagnetic resonance (EPR). The EPR technique gives better results at unpaired electron systems. The FMR technique depends on the geometry of the sample at hand. The demagnetization field is observed where the sample geometry is active. The resonance area of the sample depends on the properties of material. The FMR technique is advantageous because it does not cause damage to materials. Also, it allows a three dimensional analysis of samples. The FMR occurs at high field values while EPR occurs at low magnetic field values. Also, line-width of ferromagnetic materials is large according to paramagnetic materials. Exchange interaction energy between unpaired electron spins that contribute to the ferromagnetism causes the line narrowing. So, ferromagnetic resonance lines appear sharper than expected.

The FMR studies have been increased since the EPR was discovered in 1945 (Zavosky, 1945; Kittel, 1946, 1947, 1949, 1953, 1958; Kip, 1949; Bloembergen, 1950, 1954; Crittenden, 1953; Van Vleck, 1950; Herring, 1950; Anderson, 1953; Damon, 1953; Young, 1953; Ament, 1955; Ruderman, 1954; Reich, 1955; Kasuya, 1956; White, 1956; Macdonald, 1956; Mercereau, 1956; Walker, 1957; Yosida, 1957; Tannenwald, 1957; Jarrett, 1958; Rado, 1958; Brown, 1962; Frait, 1965; Sparks, 1969). The beginnings of theoretical and experimental studies of spectroscopic investigations of basic sciences are used such as physics, chemistry, especially nanosciences and nanostructures (Rodbell, 1964; Kooi, 1964; Bhagat, 1967, 1974; Sparks, 1970(a), 1970(b), 1970(c), 1970(d); Rachford, 1981; Dillon, 1981; Schultz, 1983; Artman, 1957, 1979; Ramesh, 1988(a), 1988(b); Fraitova, 1983(a), 1983(b), 1984; Teale, 1986; Speriosu, 1987; Vounyuk, 1991; Roy, 1992; Puszkarski, 1992; Weiss, 1955). The FMR technique can provide information on the magnetization, magnetic anisotropy, dynamic exchange/dipolar energies and relaxation times, as well as the damping in the magnetization dynamics (Wigen, 1962, 1984, 1998; De Wames, 1970; Wolfram, 1971; Yu, 1975; Frait, 1985, 1998; Rook, 1991; Bland, 1994; Patton, 1995, 1996; Skomski, 2008; Coey, 2009). This spectroscopic method/FMR have been used to magnetic properties (Celinski, 1991; Farle, 1998, 2000; Fermin, 1999; Buschow, 2004; Heinrich, 2005(a), 2005(b)), films (Özdemir, 1996, 1997), monolayers (Zakeri, 2006), ultrathin and multilayers films (Layadi, 1990(a), 1990(b), 2002, 2004; Wigen, 1993; Zhang, 1994(a), 1994(b); Farle, 2000; Platow, 1998; Anisimov, 1999; Y1ld1z, 2004; Heinrich, 2005(a); Lacheisserie, 2005; de Cos, 2006; Liua, 2012; Schäfer, 2012), the angular, the frequency (Celinski, 1997; Farle, 1998), the temperature dependence (Platow, 1998), interlayer exchange coupling (Frait, 1965, 1998; Parkin, 1990, 1991(a), 1991(b), 1994; Schreiber, 1996; Rook, 1991; Wigen, 1993; Layadi, 1990(a); Heinrich, 2005; Paul, 2005), Brillouin light scattering (BLS) (Grünberg, 1982; Cochran, 1995; Hillebrands, 2000) and sample inhomogeneities (Artman, 1957, 1979; Damon, 1963; McMichael, 1990; Arias, 1999; Wigen, 1998; Chappert, 1986; Gnatzig, 1987; Fermin, 1999) of samples. Besides using FMR to characterize magnetic 
properties, it also allows one to study the fundamental excitations and technological applications of a magnetic system (Schmool, 1998; Voges, 1998; Zianni, 1998; Grünberg, 2000, 2001; Vlasko-Vlasov, 2001; Zhai, 2003; Aktaş, 2004; Birkhäuser Verlag, 2007; Seib, 2009). The various thickness, disk array, half-metallic ferromagnetic electrodes, magnon scattering and other of some properties of samples have been studied using the FMR tehniques (Mazur, 1982; da Silva, 1993; Chikazumi, 1997; Song, 2003; Mills, 2003; Rameev, 2003(a), 2003(b), 2004(a), 2004(b); An, 2004; Ramprasad, 2004; Xu, 2004; Wojtowicz, 2005; Zakeri, 2007; Tsai, 2009; Chen, 2009). The magnetic properties of single-crystalline (Kambe, 2005; Brustolon, 2009), polycrystalline (Singh, 2006; Fan, 2010), alloy films (Sihues, 2007), temperature dependence and similar qualities have been studied electromagnetic spectroscopy techniques (Özdemir, 1998; Birlikseven, 1999(a), 1999(b); Fermin, 1999; Rameev, 2000; Aktaş, 2001; Budak, 2003; Khaibullin, 2004). The magnetic resonance techniques (EPR, FMR) have been applied to the iron oxides, permalloy nanostructure (Kuanr, 2005), clustered, thermocouple connected to the ferromagnet, thin permalloy layer and et al. (Guimarães, 1998; Spoddig, 2005; Can, 2012; Rousseau, 2012; Valenzuela, 2012; Bakker, 2012; Maciá, 2012; Dreher, 2012; Kind, 2012; Li, 2012; Estévez, 2012; Sun, 2012(a), 2012(b), 2012(c); Richard, 2012). Magneto-optic (Paz, 2012), dipolar energy contributions (Bose, 2012), nanocrystalline (Maklakov, 2012; Raita, 2012), La0.7Sro.3MnO3 films (Golosovsky, 2012), La0.67 Ba0.33Mn1-yA $\mathrm{O}_{3}$, A - Fe, Cr (Osthöver, 1998), voltage-controlled magnetic anisotropy (VCMA) and spin transfer torque ( $\mathrm{Zhu}, 2012)$ and the typical properties of the inertial resonance are investigated (Olive, 2012). The exchange bias (Backes, 2012), Q cavities for magnetic material (Beguhn, 2012), $\mathrm{MgO} / \mathrm{CoFeB} / \mathrm{Ta}$ structure (Chen, 2012), the interfacial origin of the giant magnetoresistive effect (GMR) phenomenon (Prieto, 2012), selfdemagnetization field (Hinata, 2012), $\mathrm{Fe}_{3} \mathrm{O}_{4} / \mathrm{InAs}(100)$ hybrid spintronic structures (Huang, 2012), granular films (Kakazei, 1999, 2001; Sarmiento, 2007; Krone, 2011; Kobayashi, 2012), nano-sized powdered barium $\left(\mathrm{BaFe}_{12} \mathrm{O}_{19}\right)$ and strontium $\left(\mathrm{Sr} \mathrm{Fe}{ }_{12} \mathrm{O}_{19}\right)$ hexaferrites (Korolev, 2012), Nio.7Mno.3-x CoxFe2 $\mathrm{O}_{4}$ ferrites (NiMnCo: $\mathrm{x}=0.00,0.04,0.06$, and 0.10) (Lee, 2012), thin films (Demokritov, 1996,1997; Nakai, 2002; Lindner, 2004; Aswal, 2005; Jalali-Roudsar, 2005; Cochran, 2006; Mizukami, 2007; Seemann, 2010), Ni2MnGa films (Huang, 2004), magnetic/electronic order of films (Shames, 2012), Fe1-xGd(Tb)x films (Sun, 2012), in $\varepsilon-$ $\mathrm{Al}_{0.06} \mathrm{Fe}_{1.94} \mathrm{O}_{3}$ (Yoshikiyo, 2012). $10 \mathrm{~nm}$ thick Fe/GaAs(110) film (Römer, 2012), triangular shaped permalloy rings (Ding, 2012) and Co2-Y hexagonal ferrite single rod (Bai, 2012) structures and properties have been studied by FMR tecniques (Spaldin, 2010). Biological applications (Berliner, 1981; Wallis, 2005; Gatteschi, 2006; Kopp, 2006; Fischer, 2008; Mastrogiacomo, 2010), giant magneto-impedance (Valenzuela, 2007; Park, 2007), dynamics of feromagnets (Vilasi, 2001; Rusek, 2004; Limmer, 2006; Sellmyer, 2006; Spinu, 2006; Azzerboni, 2006; Krivoruchko, 2012), magneto-optic kerr effect (Suzuki, 1997; Neudecker, 2006), Heusler alloy (HA) films (Kudryavtsev, 2007), ferrites (Kohmoto, 2007), spin polarized electrons (Rahman, 2008) and quantum mechanics (Weil, 2007) have been studied by FMR technique in generally (Hillebrands, 2002, 2003, 2006). In additional, electric and magnetic properties of pure, $\mathrm{Cu}^{2+}$ ions doped hydrogels have been studied by ESR techniques (Coşkun, 2012). 
The FMR measurements were performed in single crystals of silicon- iron, nickel-iron, nickel and hcp cobalt (Frait, 1965), thin films (Knorr, 1959; Davis, 1965; Hsia, 1981; Krebs, 1982; Maksymowich, 1983, 1985, 1992; Platow, 1998; Durusoy, 2000; Baek, 2002; Kuanr, 2004), CoCr magnetic thin films (Cofield, 1987), NiFe/FeMn thin films (Layadi, 1988), single-crystal $\mathrm{Fe} / \mathrm{Cr} / \mathrm{Fe}(100)$ sandwiches (Krebs, 1989), polycrystalline single films (Hathaway, 1981; Rezende, 1993) and ultrathin multilayers of the system $\mathrm{Au} / \mathrm{Fe} / \mathrm{Au} / \mathrm{Pd} / \mathrm{Fe}(001)$ prepared on GaAs(001) (Woltersdorf, 2004). The FMR techniques have been succesfully applied peak-topeak linewidth (Yeh, 2009; Sun, 2012), superconducting and ferromagnetic coupled structures (Richard, 2012) and thin Co films of $50 \mathrm{~nm}$ thick (Maklakov, 2012). The garnet materials (Ramesh, 1988 (a), 1988 (b)), polar magneto-optic kerr effect and brillouin light scattering measurements (Riedling, 1999), giant-magnetoresistive (GMR) multilayers (Grünberg, 1991; Borchers, 1998) and insulated multilayer film (de Cos, 2006; Lacheisserie, 2005) are the most intensely studied systems.

The technique of FMR can be applied to nano-systems (Poole, 2003; Parvatheeswara, 2006; Mills, 2006; Schmool, 2007; Vargas, 2007; Seemann, 2009; Wang, 2011; Patel, 2012; De Biasi, 2013). The FMR measurement on a square array of permalloy nanodots have been comparion a numerical simulation based on the eigenvalues of the linearized LandauLifshitz equation (Rivkin, 2007). The dynamic fluctuations of the nanoparticles and their anisotropic behaviour have been recorded with FMR signal (Owens, 2009). Ferromagnetic resonance (FMR) modes for $\mathrm{Fe}_{70} \mathrm{CO}_{30}$ magnetic nanodots of $100 \mathrm{~nm}$ in diameter in a monodomain state are studied under different in-plane and out-of-plane magnetic fields (Miyake, 2012). The FMR techniques have been accomplished applied to magnetic microwires and nanowire arrays (Adeyeye, 1997; Wegrowe, 1999, 2000; García-Miquel, 2001; Jung, 2002; Arias, 2003; Raposo, 2011; Boulle, 2011; Kraus, 2012; Klein, 2012). In additional, FMR measurements have been performed for nanocomposite samples of varying particles packing fractions with demagnetization field (Song, 2012). The ferromagnetic resonance of magnetic fluids were theoretically investigated on thermal and particles size distribution effects (Marin, 2006). The FMR applied to nanoparticles, superparamagnetic particles and catalyst particles (de Biasi, 2006; Vargas, 2007; Duraia, 2009).

In the scope of this chapter, we firstly give a detailed account of both magnetic order and their origin. The origin of magnetic orders are explained and the equations are obtained using Fig. 1 which shows rotating one electron on the table plane. Then, the dynamic equation of motion for magnetization was derived. We mentioned MR and damping terms which have consisted three terms as the Bloch-Bloembergen, the Landau-Lifshitz and the Gilbert form. We indicated electron EPR/ESR and their historical development. The information of spin Hamiltonian and $g$-tensor is given. The dispersion relations of monolayer, trilayers, five-layers and multilayer/ $n$-layers have regularly been calculated for ferromagnetic exchange-couple systems (Grünberg, 1992; Nagamine, 2005, Schmool, 1998). The theoretical FMR spectra were obtained by using the dynamic equation of motion for magnetization with the Bloch-Bloembergen type damping term. The exchange-spring (hard/soft) system which is the best of the sample for multilayer structure has been explained by using the FMR technique and equilibrium condition of energy of system. The 
FMR spectra originated from the iron/soft layers as shown in the exchange spring magnets in Fig.9. Finally, superparamagnetic/single-domain nanoparticles and their resonance are described in detail.

\section{Magnetic order}

Magnetic materials are classified as paramagnetic, ferromagnetic, ferrimagnetic, antiferromagnetic and diamagnetic to their electronic order. Magnetic orders are divided in two groups as (i) paramagnetic, ferromagnetic, ferrimagnetic, antiferromagnetic and (ii) diamagnetic. The magnetic moments in diamagnetic materials are opposite to each other as well as the moments associated with the orbiting electrons so that a zero magnetic moment $(\mu)$ is produced on macroscopic scale. In the paramagnetic materials, each atom possesses a small magnetic moment. The orientation of magnetic moment of each atom is random, the net magnetic moment of a large sample (macroscopic scale) of dipole and the magnetization vector are zero when there is no applied field.

Nanoscience, nanotechnology and nanomaterials have become a central field of scientific and technical activity. Over the last years the interest in magnetic nanostructures and their applications in various electronic devices, effective opto-electronic devices, bio-sensors, photo-detectors, solar cells, nanodevices and plasmonic structures have been increasing tremendously. This is caused by the unique properties of magnetic nanostructures and the outstanding performance of nanoscale devices. Dimension in the range of one to hundred nanometers, is called the nano regime. In recent years, nanorods, nanoparticles, quantum dots, nanocrystals etc. are in a class of nanostructures (Yalçın, 2012; Kartopu \& Yalçın, 2010; Aktaş, 2006) studied extensively. As the dimensions of nano materials decrease down to the nanometer scale, the surface of nanostructures starts to exhibit new and interesting properties mainly due to quantum size effects.

\section{Origin of magnetic moment}

The magnetization of a matter is derived by electrons moving around the nucleus of an atom. Total magnetic moment occurs when the electrons such as a disc returns around its axis consist of spin angular momentum and returns around the nucleus consist orbital angular momentum. The most of matters which have unpaired electrons have a little magnetic moment. This natural angular momentum consists of the result of charged particle return around its own axis and is called spin of the particle. The origin of spin is not known exactly, although electron is point particle the movement of an electron in an external magnetic field is similar to the movement of the disc. In other words, the origin of the spin is quantum field theoretical considerations and comes from the representations of the Poincare algebra for the elementary particles. The magnetism related to spin angular momentum, orbital angular momentum and spin-orbit interactions angular momentum. The movement of the electron around the nucleus can be considered as a current loop while electron spin is considered very small current loop which generate magnetic field. Here, orbital angular 
momentum was obtained by the result of an electron current loop around the nucleus. Thus, both it is exceeded the difficulty of understanding the magnetic moment and the magnetic moment for an electron orbiting around the nucleus is used easily. The result of orbitalangular momentum $(\vec{L})$ adapted for spin-angular momentum $(\vec{S})$ (Cullity, 1990).

One electron is rotating from left to right on the table plane as shown in Fig.1. The rotating electron creates a current $(i)$ on the circle with radius of $r$.

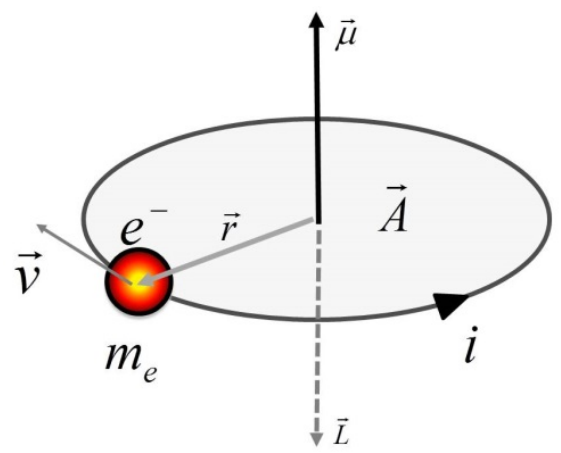

Figure 1. Schematic representation of the precession of a single electron on the table plane.

The magnetic moment of a single electron is defined as below

$$
\vec{\mu}=i \cdot \vec{A}
$$

Where, $\vec{A}$ is the circle area. The magnetic moment is written as follows by using the current $(-e=i \cdot t)$, one cycle $(2 \pi r=v \cdot t)$ and angular momentum $\left(L=m_{e} \cdot v \cdot r\right)$ definition.

$$
\vec{\mu}=-\frac{e}{2 m_{e}} \vec{L}
$$

Where $\gamma=e / 2 m_{e}$ and $\vec{L}$ is the gyromagnetic (magneto-mechanical or magneto-gyric) ratio and the orbital-angular momentum, respectively. Therefore, the magnetic moment $\vec{\mu}$ is obtained from Eq. (2) as below

$$
\vec{\mu}=-\gamma \vec{L} .
$$

The following expression is obtained when derivative of Eq.(3)

$$
d \vec{\mu}+\gamma d \vec{L}+\vec{L} d \gamma=0
$$

For our purpose, we only need to know that $\gamma$ is a constant and $d \gamma=0$. From this results, $d \vec{\mu}+\gamma d \vec{L}=0$. The derivative of time of this equation, the equation of motion for magnetic moments of an electron is found as below 


$$
\frac{1}{\gamma} \frac{d \vec{\mu}}{d t}=\frac{d \vec{L}}{d t}=\vec{\tau}
$$

This equation is related to $\vec{\tau}=d \vec{L} / d t$ in two dimensional motions on the plane and $\vec{F}=d \vec{P} / d t$ in one dimensional motion. This motion corresponds to Newton's dynamic equations. When an electron is placed in an applied magnetic field $\vec{H}$, the magnetic field will produce a torque $(\vec{\tau})$ on the magnetic moment $(\vec{\mu})$ of amount $\vec{\mu} \times \vec{H}$. The equation of motion for magnetic moment $(\vec{\mu})$ is found by equating the torque as below

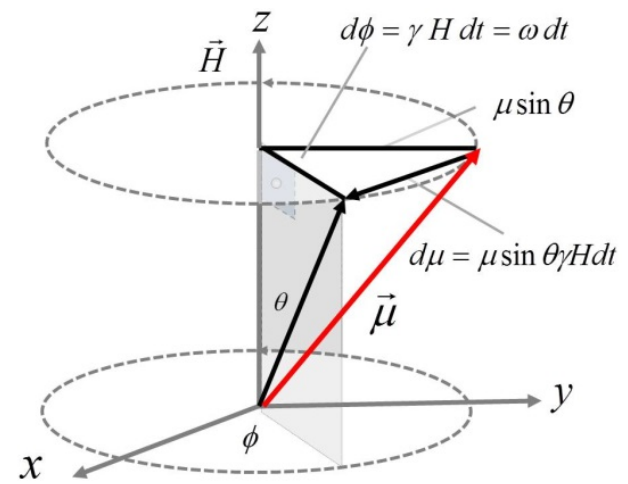

Figure 2. Schematic representation of precession of a single magnetic moment $\vec{\mu}$ in the external magnetic field around the z-axis.

$$
\frac{1}{\gamma} \frac{d \vec{\mu}}{d t}=\vec{\mu} \times \vec{H}
$$

This expression is called the equation of motion for magnetic moment $(\vec{\mu})$. The motion of magnetic moment $(\vec{\mu})$ forms a cone related to $\vec{H}$ when the angle $\theta$ of magnetic moment and external magnetic field does not change. Therefore, in time $(d t)$, the tip of the vector $\vec{\mu}$ moves an angle $(\gamma H) d t$. The magnetic moment vector make precession movement about $\vec{H}$ at a frequency of $\gamma H / 2 \pi$. This frequency, $v=\omega / 2 \pi=\gamma H / 2 \pi$, is called the Larmor frequency. In general this Larmor frequency is used this form $\omega=\gamma H$ in literature.

\section{Magnetic resonance}

Magnetic Resonance (MR) is a research branch which examines magnetic properties of matters. The magnetic properties of atom originate from electrons and nucleus. So, it is studied in two groups such as electron paramagnetic resonance (EPR)/electron spin resonance (ESR) and nuclear magnetic resonance (NMR). At ESR and NMR all of them are the sample is placed in a strong static magnetic field and subjected to an orthogonally amplitude-frequency. While EPR uses a radiation of microwave frequency in general, NMR is observed at low radio frequency range. The energy absorption occurs when radio 
frequency is equal with energy difference between electrons two levels. But, the transition must obey the selection rules. The splitting between the energy levels occurs when total angular moment of electron is different from zero. On the other hand, the splitting of energy levels has not been observed in the filled orbit. The precession motion of a paramagnetic sample in magnetic field is seen schematically in Fig. 2. If microwave field with $v$ frequency at perpendicular is applied to the static field, it comes out power absorption when precession $\left(\omega_{0}\right)$ is same with $v$-frequency. The power increases when these frequencies come near to each other and it occurs maximum occurs at point when they are equal. This behaviour is called magnetic resonance (MR).

The magnetic materials contain a large number of atomic magnetic moment in generally. Net atomic magnetic moment can be calculated by $\vec{M}=N \vec{\mu}$. Where, $N$ is the number of atomic magnetic moment in materials.

$$
\frac{1}{\gamma} \frac{d \vec{M}}{d t}=\vec{M} \times \vec{H}_{e f f}
$$

This precession movement continue indefinitely would take forever when there is no damping force. The damping term may be introduced in different ways. Indeed, since the details of the damping mechanism in a ferromagnet have not been completely resolved, different mathematical forms for the damping have been suggested. The three most common damping terms used to augment the right-hand side of Eq. (7) are as follows:

(i) The Bloch-Bloembergen form: $-\frac{\vec{M}_{\theta, \varphi}}{T_{2}}-\frac{\vec{M}_{z}-M_{0}}{T_{1}}$

(ii) The Landau-Lifshitz form: $\frac{-\lambda}{|\vec{M}|^{2}} \vec{M} \times \vec{M} \times \vec{H}$

(iii) The Gilbert form: $\frac{\alpha}{|\vec{M}|} \vec{M} \times \frac{d \vec{M}}{d t}$

Bloch-Bloembergen type damping does not converse $M$ so it is equivalent to the type of Landau-Lifshitz and the Gilbert only when $\alpha$ is small and for small excursion of $\vec{M}$. For large excursion of $M$, the magnitude of $\vec{M}$ is certainly not protected, as the damping torque is in the direction of the magnetization component in this formularization. Hence, the observation of $M$ in the switching experiments in thin films should be provide a sensitive test on the appropriate form of the damping term for ferromagnetism since $\vec{M}$ which is conserved during switching. This would suggest that the form of the Bloch-Bloembergen damping term would not be applicable for this type of experiment. The Gilbert type (Gilbert, 1955) is essentially a modification of the original form which is proposed firstly by Landau and Lifshitz (Landau \& Lifshitz, 1935). It is very important to note that the LandauLifshitz and Gilbert type of damping conserve while the Bloch-Bloembergen (Bloembergen, 
1950) type does not. Landau and Lifshitz observed that the ferromagnetic exchange forces between spins are much greater than the Zeeman forces between the spins and the magnetic fields in their formulation of the damping term. Therefore, the exchange will conserve the magnitude of $\vec{M}$. In this formulation, since the approach of $\vec{M}$ towards $\vec{H}$ is due completely to the relatively weak interaction between $\vec{M}$ and $\vec{H}$, we must require that $\lambda<<\gamma M$. In this small damping limit, the Landau-Lifshitz and the Gilbert forms are equivalence so that whether one uses one or the other is simply a matter of convenience or familiarity. However, Callen has obtained a dynamic equation by quantizing the spin waves into magnons and treating the problem quantum-mechanically (Callen, 1958). Subsequently, Fletcher, Le Craw, and Spencer have reproduced the same equation using energy consideration (Fletcher, 1960). In their reproduction, they found the mean the rate of energy transfer between the uniform precession, the spin waves (Grünberg, 1979, 1980) and the lattice.

\section{Electron paramagnetic resonance}

Stern and Gerlach (Gerlach, 1922) proved that the electron-magnetic moment of an atom in an external magnetic field originates only in certain directions in the experiment in 1922. Uhlenbek and Goudsmit found that the connection between the magnetic moment and spin angular momentum of electron (Uhlenbek, 1925), Rabi and Breit found the transition between the energy levels in oscillating magnetic field (Rabi, 1938). This also proved to be observed in the event of the first magnetic resonance. The EPR technique is said to be important of Stern-Gerlach experiment. Zavoisky observed the first peak in the electron paramagnetic resonance for $\mathrm{CuCl}_{2} 2 \mathrm{H}_{2} \mathrm{O}$ sample and recorded (Zavoisky, 1945). The most of EPR experiments were made by scientists in the United Kingdom and the United States. Important people mentioned in the experimental EPR studies; Abragam, Bleaney and Van Vleck. The historical developments of MR have been summarized by Ramsey (Ramsey, 1985). NMR experiments had been done by Purcell et al. (Purcell, 1946). Today it has been used as a tool for clinical medicine. MRI was considered as a basic tool of CT scan in 1970s. The behaviors of spin system under the external magnetic field with the gradient of spin system are known NMR tomography. This technique is used too much for medicine, clinics, diagnostic and therapeutic purposes. General structure of the EPR spectrometer consist four basic parts in general. (i) Source system (generally used in the microwave 1-100 GHz), (ii) cavity-grid system, (iii) Magnet system and (iv) detector and modulation system. EPR/ESR is subject of the MR. An atom which has free electron when it is put in magnetic field the electron's energy levels separate (Yalçın, 2003, 2007(a), 2007(b)). This separation originates from the interaction of the electrons magnetic moment with external magnetic field. Energy separating has been calculated by the following Hamiltonian.

$$
\hat{H}=g \mu_{B} \vec{H} \cdot \hat{S}
$$

It is called Zeeman Effect. If the applied magnetic field oriented z-axis energy levels are;

$$
E_{M s}=g \mu_{B} H \cdot M_{s} .
$$


Here, $g$ is the $g$-value (or Landé $g$-value) (for free electron $g_{e}=2.0023193$ and proton $\left.g_{N}=2.7896\right), \mu_{B}$ is Bohr magneton $\left(\mu_{B}=\left(e h / 4 \pi m_{e}\right)=9.2740 \times 10^{-24} J / T\right)$ and $M_{s}$ is the number of magnetic spin quantum. If the orbital angular momentum of electron is large of zero $(L>0) g$-value for free atoms is following

$$
g=1+\frac{S(S+1)-L(L+1)+J(J+1)}{2 J(J+1)} .
$$

The anisotropy of the $g$-factor is described by taking into account the spin-orbit interaction combined (Yalçın, 2004(c)). The total magnetic moment can be written at below;

$$
\mu_{\text {eff }}=g \mu_{B} \sqrt{J(J+1)}
$$

The values of orbital angular momentum of unpaired electrons for most of the radicals and radical ions are zero or nearly zero. Hence, the number of total electron angular momentum $J$ equals only the number of spin quantum $S$. So, these values are nearly 2 . For free electron $\left(M_{s}= \pm 1 / 2\right)$ and for this electron;

$$
\Delta E=E_{+1 / 2}-E_{-1 / 2}=g \mu_{B} H .
$$

When the electromagnetic radiation which frequency $v$ is applied to such an electron system;

$$
h v=g \mu_{B} H \text {. }
$$

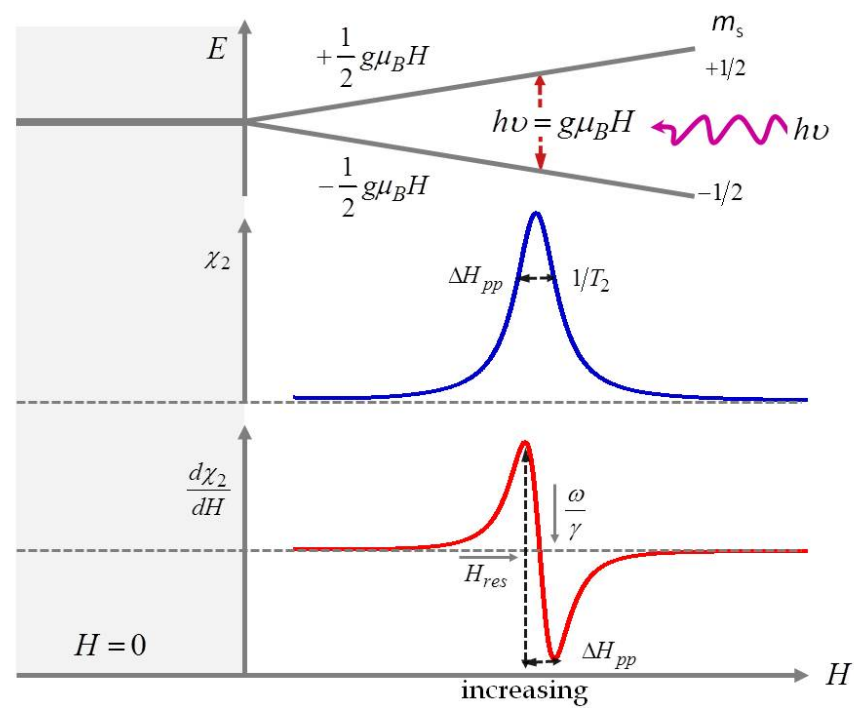

Figure 3. The energy levels and resonance of free electron at zero field and increasing applied magnetic field. In this figure, while the value of magnetic field increases, the separating between energy levels increase. Arbitrary units used in vertical axes for $\chi_{2}$ and $d \chi_{2} / d H$. 
If this equation is provided the system absorbs energy from applied electromagnetic wave (see Fig. 3). It is called resonance effect. Material absorbs energy in two different ways from applied electromagnetic wave by according to the Eq.(12). Firstly, in Eq.(12), frequency of electromagnetic wave doesn't change while the external magnetic field changes. Secondly, its opposite can be provided.

In this Fig.(3) it has been seen that magnetic susceptibility $\chi_{2}$ versus magnetic field. At the same time it is said the absorption curve. The magnetic field derivative beneath of this figure is FMR absorption spectrum $\left(d \chi_{2} / d H\right)$. Here, $\Delta_{p p}$ and $1 / T_{2}$ are linewidth, $H_{r e s}$ is resonance field, $\omega / \gamma$ is resonance frequency.

\subsection{Spin Hamiltonian}

The spin Hamiltonian is total electronic spins and nucleon spin $\vec{I}$ which have crystal lattice under the static magnetic field following;

$$
\hat{H}=\mu_{B} \vec{H} \cdot \overrightarrow{\vec{g}} \cdot \hat{S}+\hat{S} \cdot \overrightarrow{\vec{D}} \cdot \hat{S}+\vec{S} \cdot \overrightarrow{\vec{A}} \cdot \hat{I}-\mu_{N} \cdot \vec{H} \cdot \vec{g}_{N} \cdot \hat{I}+\hat{I} \cdot \overrightarrow{\vec{P}} \cdot \hat{I}
$$

$\hat{S}$ and $\vec{I}$ operators of electronic and nucleus, respectively. In this equation, first term is Zeeman effect, second one is thin layer effects, the third one is the effect of between electronic spin and nucleus-spin of ion and it is known that thin layer effects. The fourth term is the effect of nucleus with the magnetic field. The last one is quadrupole effect of nucleus. It can be added different terms in Eq.13 (Slichter, 1963).

\section{2. $\overrightarrow{\vec{g}}$ tensor}

The total magnetic moment of ion is $\vec{\mu}=g \mu_{B} \vec{J} . \vec{J}$ is the ratio of total angular momentum to Planck constant. Landé factor $g$ is depend on $\vec{S}, \vec{L}, \vec{J}$. For the base energy level if $\vec{L}$ is zero, $g$ factor is equal free electron's $g$-factor. But, $g$-factor in the exited energy levels separated from the $g$-factor of free electron. The hamiltonian for an ion which is in the magnetic field is following (Weil, 1994).

$$
\hat{H}=\mu_{B} \vec{H} \cdot\left(\hat{L}+g_{e} \hat{S}\right)+\lambda \hat{L} \cdot \hat{S}
$$

In this equation, first term is Zeeman effects, second one is spin-orbit interaction. The first order energy of ion which shows $|J, M\rangle$ and it is excepted not degenerate is seen at below.

$$
E_{J}=\left\langle J, M\left|g_{e} \mu_{B} H_{z} \hat{S}_{z}\right| J, M\right\rangle+\left\langle J, M\left|\left(\mu_{B} H_{Z}+\lambda \hat{S}_{z}\right)\right| J, M\right\rangle
$$

We can write the hamiltonian equation which uses energy equations. There is two terms in the hamiltonian equations. The first term is the independent temperature coefficient for paramagnetic for paramagnetic, the last terms are only for spin variables. If the angular moment of ion occurs because of spin, $\overrightarrow{\vec{g}}$-tensor is to be isotropic. 


\section{Ferromagnetic resonance}

The most important parameters for ferromagnet can be deduced by the ferromagnetic resonance method. FMR absorption curves may be obtained from Eq.(12) by chancing frequency or magnetic field. FMR signal can be detected by the external magnetic field and frequency such as EPR signal. The field derivative FMR absorption spectra are greater than in EPR as a generally. The linear dependence of frequency of resonance field may be calculated from $1 \mathrm{GHz}$ to $100 \mathrm{GHz}$ range in frequency spectra (L-, S-, C-, X-, K-, Q-, V-, E-, $\mathrm{W}-, \mathrm{F}-$, and D-band). The resonance frequency, relaxation, linewidth, Landé $g$-factor (spectroscopic $g$-factor), the coercive force, the anisotropy field, shape of the specimen, symmetry axes of the crystal and temperature characterized FMR spectra. The broadening of the FMR absorption line depend on the line width (so called $1 / T_{2}$ on the BlochBloembergen type damping form). The nonuniform modes are seen in the EPR signal. The nonlinear effects for FMR are shown by the relationship between the uniform precessions of magnetic moments. The paramagnetic excitation of unstable oscillation of the phonons displays magneto-elastic interaction in ferromagnetic systems. This behaviour so called magnetostriction. The FMR studies have led to the development of many micro-wave devices. These phenomenon are microwave tubes, circulators, oscillators, amplifiers, parametric frequency converters, and limiters. The resonance absorption curve of electromagnetic waves at centimeter scale by ferromagnet was first observed by Arkad'ev in 1913 (Arkad'ev, 1913) .

The sample geometry, relative orientation of the equilibrium magnetization $\vec{M}$, the applied dc magnetic field $\vec{H}$ and experimental coordinate systems are shown in Fig.4.

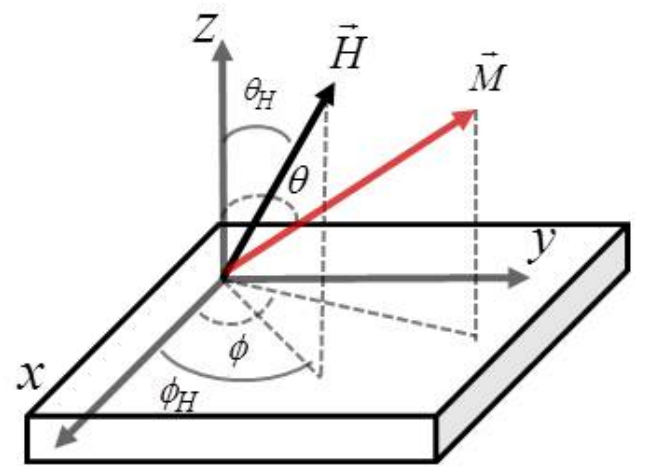

Figure 4. Sample geometries and relative orientations of equilibrium magnetization $\vec{M}$ and the $d c$ components of external magnetic field, $\vec{H}$ for thin films.

The ferromagnetic resonance data analyzed using the free energy expansion similar to that employed 


$$
\begin{aligned}
& E_{T}=E_{\mathrm{Z}}+E_{\mathrm{a}}+E_{\mathrm{d}}+E_{\mathrm{ex}}+\ldots \\
& E_{\mathrm{Z}}=-M \cdot H\left(\sin \theta \sin \theta_{H} \cos \left(\phi-\phi_{H}\right)+\cos \theta \cos \theta_{H}\right) \\
& E_{\mathrm{a}}=K_{\mathrm{u} 1} \sin ^{2} \theta+K_{\mathrm{u} 2} \sin ^{4} \theta+K_{\mathrm{u} 3} \sin ^{6} \theta \ldots \\
& E_{\mathrm{d}}=-2 \pi M^{2} \sin ^{2} \theta \\
& E_{e x}=-J S_{i} S_{j}
\end{aligned}
$$

Where, $E_{\mathrm{Z}}, E_{\mathrm{a}}, E_{\mathrm{d}}, E_{\mathrm{ex}}$ are Zeeman, magnetocrystalline anisotropy, demagnetization and ferromagnetic exchange energy. $(\theta, \phi)$ and $\left(\theta_{H}, \phi_{H}\right)$ are the angles for magnetization and applied magnetic field vector in the spherical coordinates, respectively. Magnetic anisotropy energy arises from either the interaction of electron spin magnetic moments with the lattice via spin-orbit coupling. On the other hand, anisotropy energy induced due to local atomic ordering. The $\theta$ in anisotropy energy is the angle between magnetization orientation and local easy axis of the magnetic anisotropy. $K_{\mathrm{u} 1}$ and $K_{\mathrm{u} 2}$ are energy density constants. The demagnetization field is proportional to the magnetic free pole density. The exchange energy for thin magnetic film may be neglected in generally. Because associated energies is small. But, this exchange energy are not neglected for multilayer structures. This energy occurs between the magnetic layers, so that this energy called interlayer exchange energy. This expression is seen at the end of this subject in details. $K_{\text {eff }}=\pi M^{2}+K_{U}$ is the effective uniaxial anisotropy term and $K_{u}$ takes into account some additional second-order uniaxial anisotropy and $H_{\text {eff }}=2 \pi M_{S}+\left(2 K_{u} / M_{s}\right)$ is the effective field for a single magnetic films. The equilibrium values of polar angles $\theta$ for the magnetization vector $\vec{M}$ are obtained from static equilibrium conditions. $E_{\theta}, E_{\phi}, E_{\theta \theta}$ and $E_{\phi \phi}$ can be easily calculated using the Eq. (16). Neglecting the damping term one can write the equation of motion for the magnetization vector $\vec{M}$ as

$$
\frac{1}{\gamma} \frac{d \vec{M}}{d t}=\vec{M} \times \vec{H}_{e f f}
$$

Here the $\vec{H}_{\text {eff }}$ is the effective magnetic field that includes the applied magnetic field and the internal field due to the anisotropy energy. The dynamic equation of motion for magnetization with the Bloch-Bloembergen type damping term is given as Eq.(18).

$$
\frac{1}{\gamma} \frac{d \vec{M}}{d t}=\vec{M} \times \vec{H}_{e f f}-\frac{\vec{M}-\delta_{i z} M_{0}}{T} .
$$

Here, $T=\left(T_{2}, T_{2}, T_{1}\right)$ represents both transverse (for $M_{x}$ and $M_{y}$ components) and the longitudinal (for $M_{z}$ components) relaxation times of the magnetization. That is, $T_{1}$ is the spin-lattice relaxation time, $T_{2}$ is the spin-spin relaxation time, and $\delta_{i z}=(0,0,1)$ for $(\mathrm{x}, \mathrm{y}, \mathrm{z})$ projections of the magnetization. In the spherical coordinates the Bloch-Bloembergen equation can be written as below; 


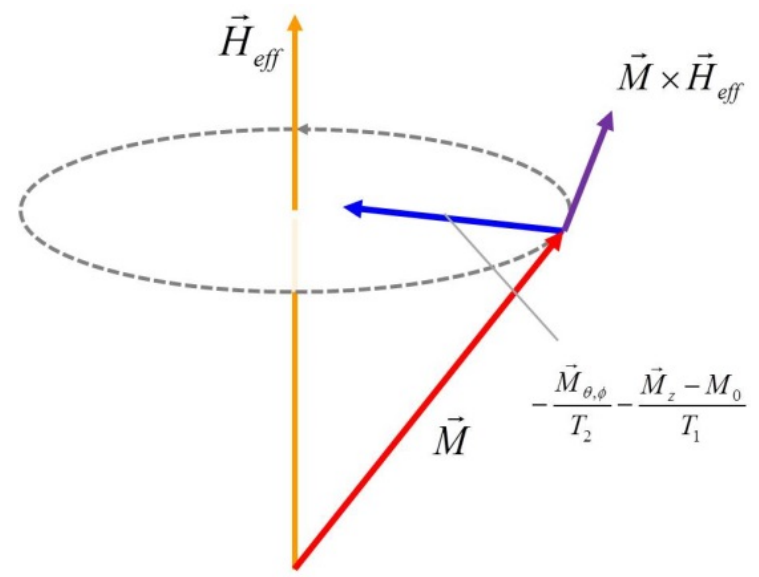

Figure 5. Damped precession of a magnetic moment $\vec{M}$ toward the effective magnetic field $\vec{H}_{\text {eff }}$ according to the Bloch-Bloembergen type equation (Aktaş, 1993, 1994; Yalçın, 2008(a)).

$$
\frac{1}{\gamma} \frac{d \vec{M}}{d t}=\frac{\vec{M}}{|\vec{M}|} \times \vec{\nabla} E-\frac{\vec{M}_{\theta, \phi}}{\gamma T_{2}}-\frac{\vec{M}_{z}-M_{0}}{\gamma T_{1}} .
$$

Where, the torque is obtained from the energy density through the expression

$$
\vec{\nabla} E=-\left(\frac{\partial E}{\partial \theta}\right) \hat{e}_{\phi}+\frac{1}{\sin \theta}\left(\frac{\partial E}{\partial \phi}\right) \hat{e}_{\theta} .
$$

For a small deviation from the equilibrium orientation, the magnetization vector $\vec{M}_{\text {can be }}$ approximated by

$$
\vec{M}=M_{s} \hat{e}_{r}+m_{\theta} \hat{e}_{\theta}+m_{\phi} \hat{e}_{\phi}
$$

Where the dynamic transverse components are assumed to be sufficiently small and can be given as

$$
\begin{aligned}
& m_{\theta}(z, t)=m_{\theta}^{0} \exp i(\omega t \pm k z) \\
& m_{\phi}(z, t)=m_{\phi}^{0} \exp i(\omega t \pm k z)
\end{aligned}
$$

Dispersion relation for films can be derived by using these solutions (Eq.(22)) in Eqs. (19) and (20). On the other hand, the eigen frequency of thin films mode is determined by the static effective field and can be derived directly from the total free energy for magnetic system/ferromagnet. It is given by the second derivatives of the total energy with respect to the $\theta$ and $\phi$ (Smit, 1955; Artman, 1957; Wigen, 1984, 1988, 1992; Baseglia, 1988; Layadi, 1990; Farle, 1998). The matrices form for $m_{\theta}$ and $m_{\phi}$ is calculated using the Eq.(19) with Eq.(20, 21, 22). 


$$
\begin{aligned}
& \left(\begin{array}{cc}
i \frac{\omega}{\gamma}+\frac{1}{\gamma T_{2}}+\frac{E_{\theta \phi}}{M_{s} \sin \theta} & \frac{E_{\phi \phi}}{M_{s} \sin ^{2} \theta} \\
-\frac{E_{\theta \theta}}{M_{s}} & i \frac{\omega}{\gamma}+\frac{1}{\gamma T_{2}}-\frac{E_{\theta \phi}}{M_{s} \sin \theta}
\end{array}\right) \cdot\left(\begin{array}{l}
m_{\theta} \\
m_{\phi}
\end{array}\right)=0 \\
& \left(\frac{\omega}{\gamma}\right)^{2}=\frac{1}{M^{2} \sin ^{2} \theta}\left(E_{\theta \theta} E_{\phi \phi}-E_{\theta \phi}^{2}\right)+\left(\frac{1}{\gamma T_{2}}\right)^{2}
\end{aligned}
$$

Here $(\omega / \gamma)=g \mu_{B} H$ is the Larmour frequency of the magnetization in the external $d c$ effective magnetic field. This dispersion relation can be related as the angular momentum analogue to be linear momentum oscillator described $\omega=\sqrt{\kappa / \mu_{i}}$. Here, restoring force constant $\kappa$ is the second derivative of the potential part in the energy of system $\kappa=E_{x x}$. The inverse mass $\mu_{i}^{-1}$ is given by the second derivative of the kinetic part in the energy with respect to linear momentum $\mu_{i}^{-1}=E_{p p}$. The restoring constant in this chapter corresponds to $E_{\theta \theta}$. The inverse mass is proportional to $E_{\phi \phi}$. The $E_{\theta \phi}$ arises when the coordinate system is not parallel to the symmetry and last term originated from relaxation term in Eq.(23) (Sparks, 1964; Morrish, 1965; Vittoria, 1993; Gurevich, 1996; Chikazumi, 1997)

The power absorption from radio frequency $(r f)$ field in a unit volume of sample is given by

$$
P=\frac{1}{2} \omega \chi_{2} h_{1}^{2}
$$

where $\omega_{\text {is }}$ the microwave frequency, $h_{1}$ is the amplitude of the magnetic field component and $\chi_{2}$ is the imaginary part of the high-frequency susceptibility. The field derivative FMR absorption spectrum is proportional to $d \chi_{2} / d H$ and the magnetic susceptibility $\chi$ is given as

$$
\chi=4 \pi\left(\frac{m_{\phi}}{h_{\phi}}\right)_{\phi=0}
$$

The theoretical absorption curves are obtained by using the imaginary part of the high frequency magnetic susceptibility as a function of applied field (Öner, 1997; Min, 2006; Cullity, 2009)

$$
\chi=\chi_{1}+i \chi_{2}=\frac{4 \pi M_{s}\left(\frac{E_{\theta \theta}}{M_{s}}\right)\left(\left(\frac{\omega_{0}}{\gamma}\right)^{2}-\left(\frac{\omega}{\gamma}\right)^{2}+i\left(\frac{2 \omega}{\gamma^{2} T_{2}}\right)\right)}{\left(\left(\frac{\omega_{0}}{\gamma}\right)^{2}-\left(\frac{\omega}{\gamma}\right)^{2}\right)^{2}+\left(\frac{2 \omega}{\gamma^{2} T_{2}}\right)^{2}}
$$

The dispersion relation can be derived by substituting Eq.(16) into Eq. (23) (Aktaş, 1997; Yalçın, 2004(a), 2004(b), 2008(a); Güner, 2006; Kharmouche, 2007; Stashkevich, 2009) 


$$
\left(\frac{\omega}{\gamma}\right)^{2}=\left[H \cos \left(\theta-\theta_{H}\right)+H_{e f f} \cos (2 \theta)\right] \cdot\left[H \cos \left(\theta-\theta_{H}\right)+H_{e f f} \cos ^{2} \theta\right]+\left(\frac{1}{\gamma T_{2}}\right)^{2}
$$

here, $\omega_{0}=2 \pi v$ is the circular frequency of the EPR spectrometer. Fitting Eq.(27) with experimental results of the FMR measurement at different out-of-plane-angle $\left(\theta_{H}\right)$, the values for the effective magnetization can be obtained.

Figure 6 uses of both experimental and theoretical coordinate systems for the nanowire sample geometry. Equilibrium magnetization $\vec{M}$ and dc-magnetic field $\vec{H}$ are shown in this figure and also the geometric factor and hexagonal nanowire array presentation of nanowire are displayed. The ferromagnetic resonance theory has been developed for thin films applied to nanowires with the help of the following Fig.6. The effective uniaxial anisotropy term for nanowire arrays films $K_{\text {eff }}=\pi M^{2}(1-3 P)+K_{U}$ is written in this manner for arrayed nanowires. The first term in the $K_{\text {eff }}$ is due to the magnetostatic energy of perpendicularly-arrayed NWs (Dubowik, 1996; Encinas-Oropesa, 2001; Demand, 2002; Yalçın, 2004(a); Kartopu, 2009, 2010, 2011(a)) and constant with the symmetry axis along wire direction. The second term in the $K_{\text {eff }}$ is packing factor for a perfectly ordered hcp NW arrays. The packing factor is defined as $P=(\pi / 2 \sqrt{3})(d / r)^{2}$. The packing factor $(P)$ of nanowires increases, nanowire diameter increases, the preferential orientation of the easy direction of magnetization changes from the parallel to the perpendicular direction to the wire axis (Kartopu, 2011(a)). As further, the effective uniaxial anisotropy ( $\left.K_{e f f}\right)$ for a perfectly ordered hcp NWs should decrease linearly with increasing packing factor. $H_{\text {eff }}=2 \pi M_{S}(1-3 P)+\left(2 K_{u} / M_{S}\right)$, which is the effective anisotropy field derived from the total magnetic anisotropy energy of NWs Eq. (16). The values for total magnetization have been obtained by fitting $H_{\text {eff }}$ with experimental results of FMR measurements at different angles $\left(\theta_{H}\right)$ of external field $\vec{H}$. The experimental spectra are proportional to the derivative of the absorbed power with respect to the applied field which is also proportional to the imaginary part of the magnetic susceptibility.

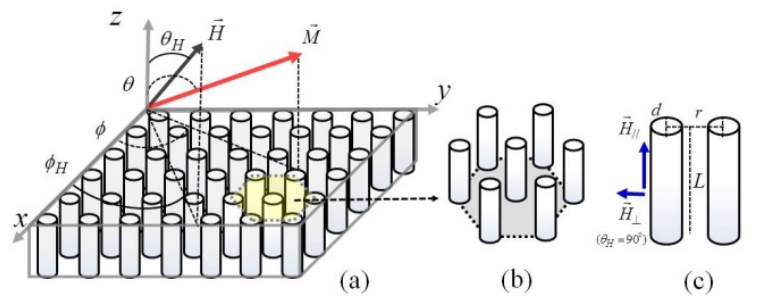

Figure 6. (a) Schematic representation of the cobalt nanowires and the relative orientation of the equilibrium magnetization $\boldsymbol{M}$ and the dc component of the external magnetic field $\boldsymbol{H}$, for the FMR experiments and their theoretical calculations. (b) Hexagonal NW array exhibiting a total of seven wires and the dashed lines bottom of the seven wires indicate the six fold symmetry. (c) Sample parameters used in the packing factors $P$ calculation. 
The experimental data were analyzed by using magnetic energy density for a system consisting of $n$ magnetic layers with saturation magnetization $M_{s}$ and layer thickness $t_{i}$. The magnetic energy density for the nanoscale multilayer structures the energy per unit surface area can be written as below

$$
E=-\left(\begin{array}{l}
\sum_{i=1}^{n} t_{i} M_{s} H\left(\sin \theta_{i} \sin \theta_{H} \cos \left(\phi_{i}-\phi_{H}\right)+\cos \theta_{i} \cos \theta_{H}\right)+ \\
\sum_{i=1}^{n} t_{i} K_{a, i} \sin ^{2} \phi_{i} \cos ^{2} \theta_{i}+\sum_{i=1}^{n} t_{i} K_{b, i} \sin ^{4} \phi_{i} \cos ^{4} \theta_{i}+ \\
\sum_{i=1}^{n-1} A_{i, i \mp 1}\left(\sin \theta_{i} \sin \theta_{i \mp 1} \cos \left(\phi_{i \mp 1}-\phi_{i}\right)+\cos \theta_{i} \cos \theta_{i \mp 1}\right)+ \\
\sum_{i=1}^{n-1} B_{i, i \mp 1}\left(\sin \theta_{i} \sin \theta_{i \mp 1} \cos \left(\phi_{i \mp 1}-\phi_{i}\right)+\cos \theta_{i} \cos \theta_{i \mp 1}\right)^{2}
\end{array}\right)
$$

Where, $\theta_{i}$ is the polar angle of the magnetization $M_{s}$ to the z-axis and $\varphi_{i}$ is the azimuth angle to the $x$-axis in the film plane. The first term is the Zeeman energy. The second and third terms correspond to first and second order magnetocrystalline energy with respectively. These energies due to the demagnetization field and any induced perpendicular anisotropy energy. On the other hand, these energies qualitatively have the same angular dependence with respect to the film normal. The second order magnetocrystalline energy term can be neglected for most of the ferromagnetic systems. The last two terms corresponds to bilinear and biquadratic interactions of ferromagnetic layers through nonmagnetic spacer via conduction energies. $A_{i, i \mp 1}$ and $B_{i, i \mp 1}$ are bilinear and biquadratic coupling constants, respectively. The bilinear exchange interaction can be written from Eq.16. $A_{i, i \mp 1}$ can be either negative and positive depending on antiferromagnetic and ferromagnetic interactions, respectively. The antiparallel/perpendicular and parallel alignments of magnetization of nearest neighboring layers are energetically favorable for a negative/positive value of $B_{i, i \neq 1}$. Biquadratic interaction for spin systems have been analysed for Ising system in detail (Chen, 1973; Erdem, 2001). The biquadratic term is smaller than the bilinear interaction term. Therefore, it can be neglected for most of the ferromagnetic systems. The indirect exchange energy depends on spacer thickness and even shows oscillatory behavior with spacer thickness (Ruderman, 1954; Yosida, 1957; Parkin, 1990, 1991(a), 19901(b), 1994). The current literature on single ultrathin films and multilayers is given in below at table (Layadi, 1990(a), 1990(b); Wigen, 1992; Zhang, 1994(a), 1994 (b); Goryunov, 1995; Ando, 1997; Farle; 1998; Platow, 1998; Schmool, 1998; Lindner, 2003; Sklyuyev, 2009; Topkaya, 2010; Erkovan, 2011).

This type exchange-coupling system is located in an external magnetic field, the magnetic moment in each layer. The suitable theoretical expression may be derived in order to deduce magnetic parameter for $a c$ susceptibility. The equation of precession motion for magnetization of the $i^{\text {th }}$ layer in the spherical coordinates with the Bloch-Bloembergen type relaxation term can be written as

$$
\frac{1}{\gamma} \frac{d \vec{M}}{d t}=\frac{1}{t_{i}} \frac{\vec{M}}{M_{i, s}} \times \vec{\nabla}_{M_{i}} E-\frac{\vec{M}_{\theta i, \varphi i}}{\gamma T_{2}}-\frac{\vec{M}_{z, i}-M_{i, s}}{\gamma T_{1}} . \quad(i=1, n)
$$


The matrices form for $m_{\theta, i-1}, m_{\phi, i-1}, m_{\theta, i}, m_{\phi, i}, m_{\theta, i+1}$ and $m_{\phi, i+1}$ of each magnetic layers calculated using the Eq.(29) with Eq.(20, 21,28).

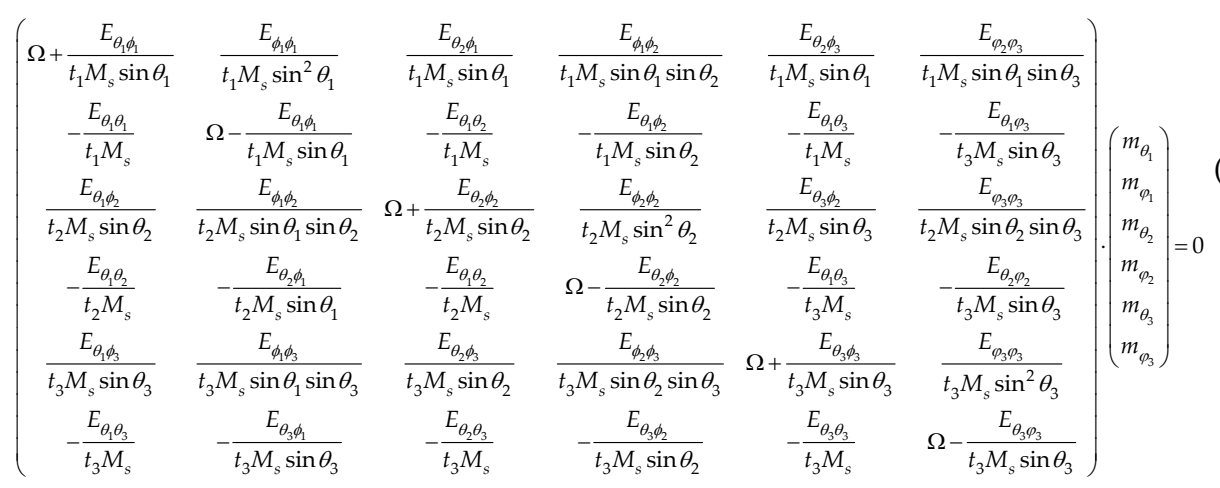

1, 2, 3 number in this Eq.(30) corresponds to $i-1, i$ and $i+1$, respectively. Here $\Omega=i\left(\frac{\omega}{\gamma}\right)+\frac{1}{\gamma T_{2}}$. Then dispersion relation for ferromagnetic exchange-coupled $n$-layers has been calculated using the $(2 \mathrm{n} \times 2 \mathrm{n})$ matrix on the left-hand side of Eq. (30) in below in detail.

$$
\left(\frac{\omega}{\gamma}\right)^{2 n}+C_{(2 n-2) / 2}\left(\frac{\omega}{\gamma}\right)^{2 n-2}+\ldots+C_{1}\left(\frac{\omega}{\gamma}\right)^{2}+C_{0}=0
$$

Here, $n$ is the number of ferromagnetic layer. $C_{0}, C_{1}, \ldots$ etc. are constant related to $t_{i}, M_{s}, E_{\theta i \theta i}, E_{\varphi i \varphi i}, E_{\theta i \varphi i}, \sin \theta_{i}$ and $\sin ^{2} \theta_{i}$. The dispersion relations for monolayer, trilayers, five-layers obtained from the Eq.(31). For tri-layers detail information are seen in ref. (Zhang, 1994(a); Schmool, 1998; Lindner, 2003). It is given that the dispersion relation for monolayer, trilayers, five-layers and multilayers/n-layers in Fig. 7.

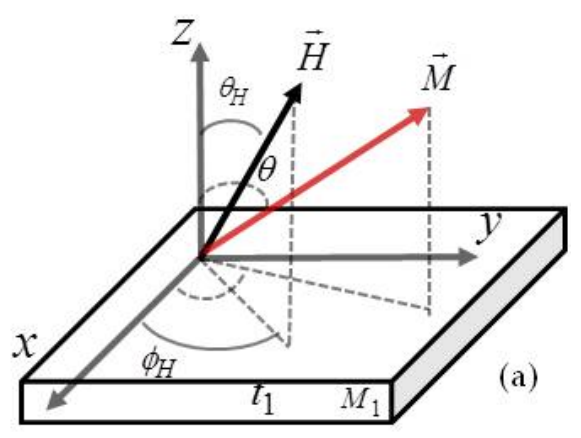

$$
\begin{aligned}
& \text { monolayer } \\
& \left(\frac{\omega}{\gamma}\right)^{2}+C_{0}=0
\end{aligned}
$$



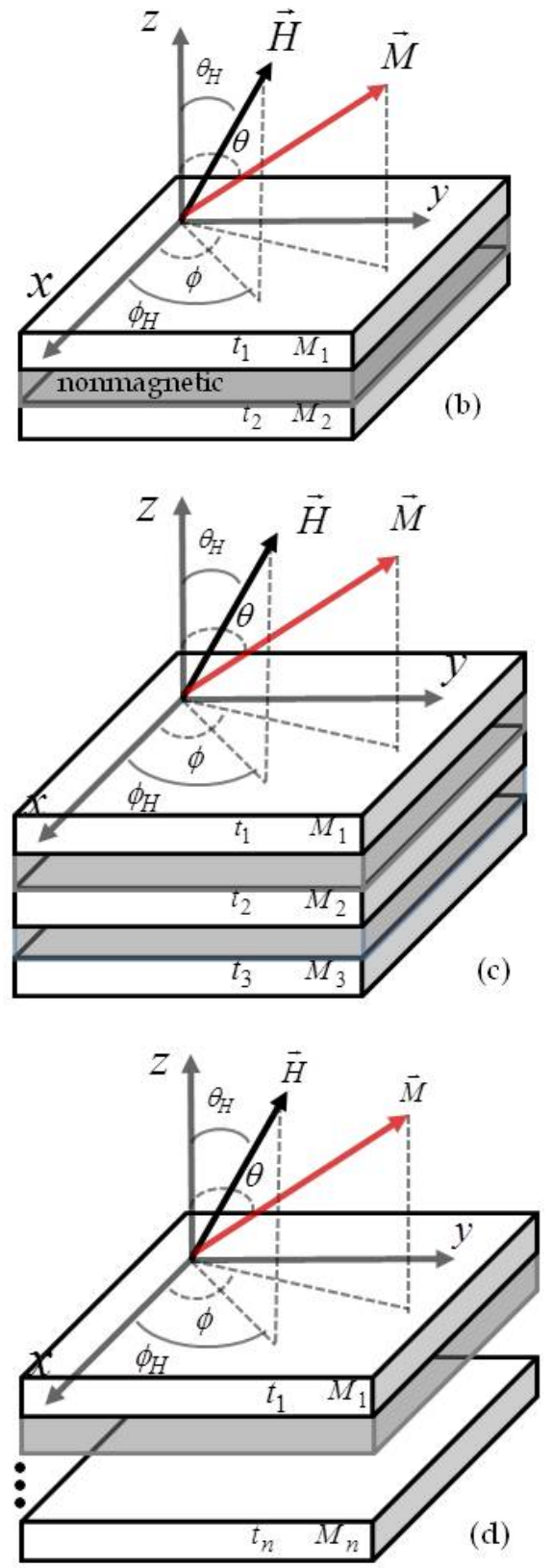

\section{trilayers}

(one nonmagnetic and two magnetic)

$$
\left(\frac{\omega}{\gamma}\right)^{4}+C_{1}\left(\frac{\omega}{\gamma}\right)^{2}+C_{0}=0
$$

\section{five-layers}

(three magnetic and two nonmagnetic layer)

$$
\left(\frac{\omega}{\gamma}\right)^{6}+C_{2}\left(\frac{\omega}{\gamma}\right)^{4}+C_{1}\left(\frac{\omega}{\gamma}\right)^{2}+C_{0}=0
$$

$$
\text { multilayer/n-layers }
$$

(n magnetic and n-1 nonmagnetic layer)

$$
\begin{aligned}
\left(\frac{\omega}{\gamma}\right)^{2 n}+C_{(2 n-2) / 2}\left(\frac{\omega}{\gamma}\right)^{2 n-2} & +\ldots \\
& +C_{1}\left(\frac{\omega}{\gamma}\right)^{2}+C_{0}=0
\end{aligned}
$$

Figure 7. Schematic representation of the (a) one layer, (b) three layer, (c) five layer and (d) n magnetic layer and their relative orientation of the equilibrium magnetization $\vec{M}$ and the dc component of the external magnetic field $\vec{H}$ for the FMR experiments and their theoretical calculations. 


\section{Example: Exchange spring (hard/soft) behaviour}

The Bloch wall, Néel line and magnetization vortex are well known properties for magnetic domain in magnetic systems. The multilayer structures are ordered layer by layer. The best of the sample for multilayer structure are exchange-spring systems. The equilibrium magnetic properties of nano-structured exchange-spring magnets may be studied in detail for some selected magnetic systems. The exchange systems are oriented from the exchange coupling between ferromagnetic and antiferromagnetic films or between two ferromagnetic films. This type structure has been extensively studied since the phenomenon was discovered (Meiklejohn, 1956, 1957). Kneller and Hawing have been used firstly the "exchange-spring" expression (Kneller, 1991). Spring magnet films consist of hard and soft layers that are coupled at the interfaces due to strong exchange coupling between relatively soft and hard layers. The soft magnet provides a high magnetic saturation, whereas the magnetically hard material provides a high coercive field. Skomski and Coey explored the theory of exchanged coupled films and predicted that a huge energy about three times of commercially available permanent magnets (120 MGOe) can be induced (Skomski, 1993; Coey, 1997). The magnetic reversal proceeds via a twisting of the magnetization only in the soft layer after saturating hard layers, if a reverse magnetic field that is higher than exchange field is applied. The spins are sufficiently closed to the interface are pinned by the hard layer, while those in deep region of soft layer rotate up to some extent to follow the applied field (Szlaferek, 2004). To be more specific, the angle of the rotation depends on the distance to the hard layer. That is the angle of rotating in a spiral spin structure similar to that of a Bloch domain wall. If the applied field is removed, the soft spins rotate back into alignment with the hard layer.

The general expression of the free energy for exchange interaction spring materials at film $\left(\theta_{i, i \pm 1}=\pi / 2\right.$ and $\left.\theta_{H}=\pi / 2\right)$ plane in spherical coordinate system as below.

$$
\begin{aligned}
E= & -\sum_{i=1}^{N} \vec{H} \cdot \vec{M}_{i}-\sum_{i=1}^{N} K_{a, i} \cos ^{2} \phi_{i}-\sum_{i=1}^{N} K_{b, i} \cos ^{4} \phi_{i} \\
& -\sum_{i=1}^{N-1} \frac{A_{i, i \mp 1}}{t^{2}} \cos \left(\phi_{i \mp 1}-\phi_{i}\right)-\sum_{i=1}^{N-1} \frac{B_{i, i \mp 1}}{t^{2}} \cos \left(\phi_{i \mp 1}-\phi_{i}\right)^{2}
\end{aligned}
$$

The expression is obtained as following using $\phi_{i} \rightarrow \phi_{i}^{\prime}$ for magnetization's equilibrium orientations of each layer at a state of equilibrium under the external magnetic field.

$$
\begin{aligned}
& \tan \phi_{i}^{\prime}= \\
& \frac{H M_{i} t^{2} \sin \phi_{H}+A_{i, i \mp 1} \sin \phi_{i \mp 1}+2 B_{i, i \mp 1} \sin \phi_{i \mp 1} \cos \left(\phi_{i}-\phi_{i \mp 1}\right)}{H M_{i} t^{2} \cos \phi_{H}+2 t^{2} K_{a, i} \cos \phi_{i}+4 t^{2} K_{b, i} \cos ^{3} \phi_{i}+A_{i, i \mp 1} \cos \phi_{i \mp 1}+2 B_{i, i \mp 1} \cos \phi_{i \mp 1} \cos \left(\phi_{i}-\phi_{i \mp 1}\right)}
\end{aligned}
$$

In this example, second-order anisotropy term $\left(K_{b, i}=0\right)$ and biquadratic interaction constant $\left(B_{i, i \pm 1}=0\right)$ considered and the result obtained show as following as adapted with 
spring magnets $\mathrm{SmCo}($ hard)/Fe(soft). For theoretical analysis, the exchange-spring magnet $\mathrm{SmCo} / \mathrm{Fe}$ is divided into subatomic multi-layers $(d=2 \AA)$, and the spins in each layer are characterized by the average magnetization $M_{i}$, and the uniaxial anisotropy constant $K_{i}$, (Fig.8).

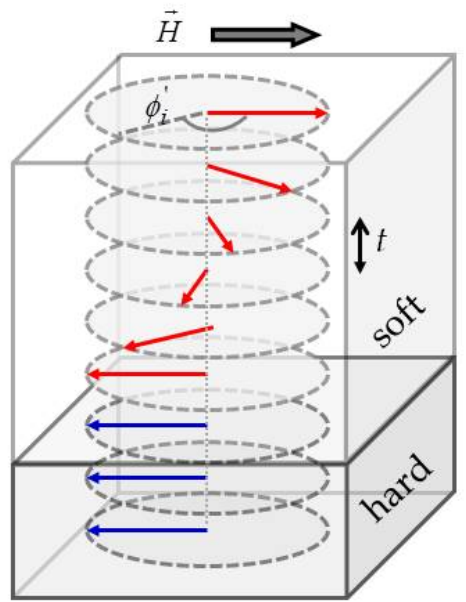

Figure 8. Schematic illustration of phases of exchange spring magnets.

Sublayers are coupled by an exchange constant $A_{i, i+1}$ (Astalos, 1998; Fullerton, 1998, 1999; Jiang, 1999, 2002, 2005; Grimsditch, 1999; Scholz, 2000; Hellwig, 2000; Pollmann, 2001; Dumesnil, 2002). $\phi_{i}$ is the angle formed by the magnetization of the $i$ th plane with the inplane (where the external field is always perpendicular to the film normal) easy axis of the hard layer (Y1ld1z, 2004(a), 2004(b)). The FMR spectra for exchange-spring magnet of

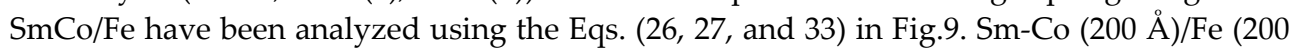
$\AA$ and $100 \AA$ ) bilayers have been grown on epitaxial $200 \AA \mathrm{Cr}(211)$ buffer layer on single crystal $\mathrm{MgO}(110)$ substrates by magnetron sputtering technique (Wüchner, 1997). To prevent oxidation $\mathrm{Sm}-\mathrm{Co} / \mathrm{Fe}$ film was coated with a $100 \AA$ thick $\mathrm{Cr}$ layer. The FMR spectra for exchange-spring magnets of $200 \AA$ and $100 \AA$ Fe samples for different angles of the applied magnetic field in the film plane are presented in Fig.9.

There are three peaks that are one of them corresponds to the bulk mode and the remaining to the surface modes for $200 \AA$ Fe sample. For more information about the FMR studies exchange spring magnets look at the ref. (Yildiz, 2004(a), 2004(b) ). Exchange-spring coupled magnets are promising systems for applications in perpendicular magnetic data recordingstorage devices and permanent magnet (Schrefl, 1993(a), 1993(b),1998, 2002; Mibu, 1997, 1998). 


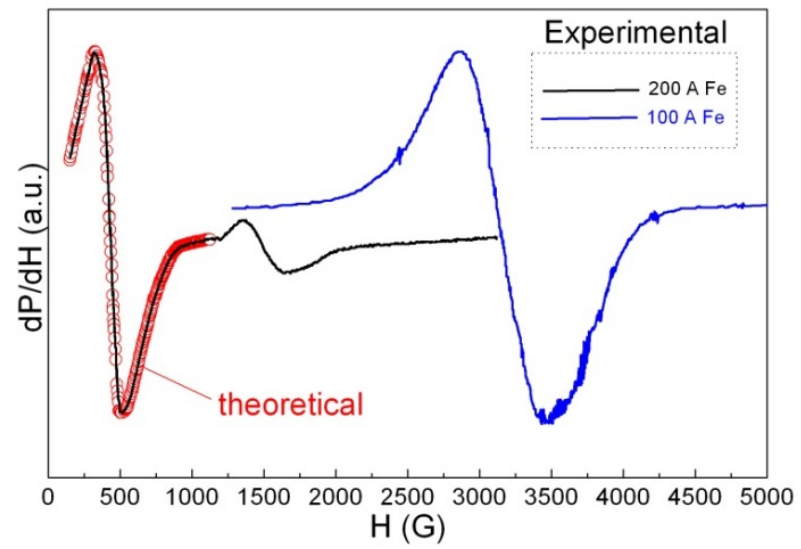

Figure 9. FMR spectra for $\operatorname{SmCo}(200 \AA) / \mathrm{Fe}(200 \AA)$ (black line) and $\mathrm{SmCo}(200 \AA) / \mathrm{Fe}(100 \AA$ ) (blue line) samples. These FMR spectra originated from the iron/soft layers.

\section{Superparamagnetic resonance}

Magnetic nanoparticles have been steadily interested in science and nanotechnology. As the dimensions of magnetic nanoparticles decrease to the nanometer scale, these nanoparticles start to exhibit new and interesting physical properties mainly due to quantum size effects (Yalçın, 2004(a), 2008(b), 2012). A single domain particle is commonly referred to as superparamagnetic (Held, 2001; Diaz, 2002; Fonseca, 2002). The superparamagnetic/singledomain nanoparticles are important for non surgical interfere of human body. Even the intrinsic physical characteristics of nanoparticles are observed to change drastically compared to their macroscopic counterparts. Stoner-Wohlfarth (Stoner, 1948) and Heisenberg model (Heisenberg, 1928) to describe the fine structure were firstly used in detail. A simple (Bakuzis, 2004) and the first atomic-scale models of the ferrimagnetic and heterogeneous systems in which the exchange energy plays a central role in determining the magnetization of the NPs, were studied (Kodama, 1996, 1999; Kodama \& Berkowitz, 1999). Superparamagnetic resonance (SPR) studies of fine magnetic nanoparticles is calculated a correlation between the line-width and the resonance field for superparamagnetic structures (Berger, 1997, 1998, 2000(a), 2000(b), 2001; Kliava, 1999). The correlation of the line-width and the resonance field is calculated from Bloch-Bloembergen equation of motion for magnetization. The SPR spectra, line width and resonance field may be analyzed by using the Eq.(34) in below. The equation of motion for magnetization with Bloch-Bloembergen type relaxation term for FMR adapted for superparamagnetic structures from Eqs.(18) and (19) in below.

$$
\chi_{2}(H)=\frac{1}{\pi} \frac{\Delta_{H}\left(\Delta_{H}^{2}+H^{2}+H_{r}^{2}\right)}{\left(\Delta_{H}^{2}+\left(H-H_{r}\right)^{2}\right) \cdot\left(\Delta_{H}^{2}+\left(H+H_{r}\right)^{2}\right)}
$$


Here, $\Delta_{H}=1 / \gamma T_{2}, H_{r}=-(\omega / \gamma)$. This equation for SPR system so called modified Bloch for fine particle magnets. The SPR microwave absorption is proportional to the imaginary part of the dynamic susceptibility. The line shape and resonance field for superparamagnet is obtained. The temperature evolution for the SPR line-width for nanoparticles can be calculated by $\Delta_{H}=\Delta_{T} L(x)$. In this expression $\Delta_{T}$ is a saturation line-width at a temperature $\mathrm{T}, L(x)=\operatorname{coth}(x)-(1 / x)$ is the Langevin function with $x=M V H_{e f f} / k_{B} T, V$ is the particle volume. The superparamagnetic (Chastellain, 2004; Dormer, 2005; Hamoudeh, 2007), coreshell nanoparticles and nanocrystalline nanoparticles (Woods, 2001; Wiekhorst, 2003; Tartaj, 2004) have been performed for possible biological applications (Sun,2005; Zhang, 2008). In additional, superparamagnetic nanoparticles have been used for hydrogels, memory effects and electronic devices (Raikher, 2003; Sasaki, 2005; Heim, 2007).

\section{Result and discussions}

The EPR, FMR and SPR signals have been observed in Fig.10. The EPR signal has reached approaching peak level about $3000 \mathrm{G}$ as seeing at Fig.10. It's symmetric and line width are narrower than resonance field, in generally. If EPR samples show crystallization, resonance field value starts to change. The EPR signal can be observed at lower temperature about $3000 \mathrm{G}$ and the signal can show crystalline property. The signal is observed in two different areas at FMR spectra as the magnetic field is parallel and perpendicular to the film. The FMR spectra are observed at low field when the magnetic field is parallel to the film, in generally. On the other hand, the FMR spectra are observed at highest field when the magnetic field is perpendicular to the film. For other conditions FMR signals are observed between these two conditions for thin films. FMR spectra can be seen a wide range of field so as to the thin films are full.

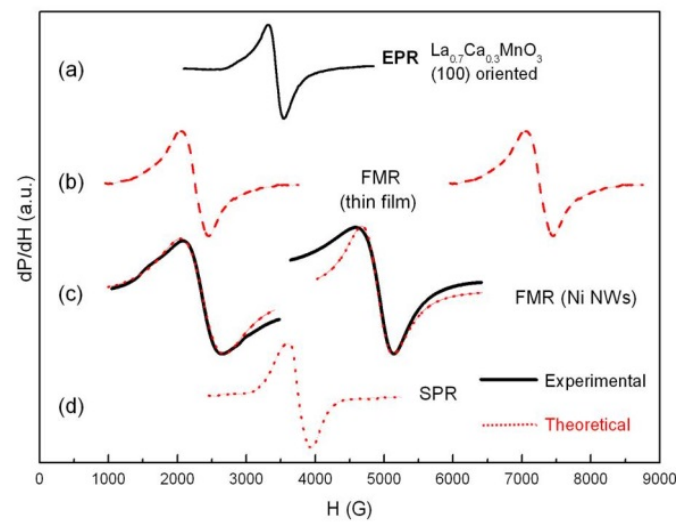

Figure 10. (a) The EPR/ESR experimental signal for La0.7Ca0.3 $\mathrm{MnO}_{3}$ samples at room temperature (see, Kartopu, 2011 (b)). (b) Theoretical FMR spectra calculated from Eq. (26) with Eq.(27) at parallel ( $\left.\theta=90^{\circ} ; 2000 \mathrm{G}\right)$ and perpendicular $\left(\theta=0^{\circ} ; \sim 7000 \mathrm{G}\right)$ position of OPG case. (c) The theoretical (reddot line in online) and experimental FMR spectra for Ni NWs $(P=29,6 ; L=0,8 \mu \mathrm{m}, \tau=13$ ) (see for detail, Kartopu, 2011 (a)). (d) The theoretical SPR signal for superparamagnet by Eq.(34) at room temperature. 
FMR spectra are similar to thin films at nanowire samples. In case of occupancy rate is that as the theoretical $P<33 \%$ for Nickel (Ni) it behaviors like thin film. But, in case of occupancy rate is that as the theoretical $P<33 \%$ it behaviors different from thin film. This situation is clearly visible from $H_{e f f}=2 \pi M_{S}(1-3 P)+\left(2 K_{u} / M_{s}\right)$. If the occupancy rate is $P<33 \%$ sample's signals show the opposite behavior according to thin film FMR signals. Look at for more information (Kartopu, 2011 (a)). This is perceived as changes the direction of the easy axis. The changes of easy axes depend on magnetization (Terry, 1917) and porosity (Kartopu, 2011 (a)) for magnetic materials/transition elements. The SPR signal is similar to EPR signal. SPR peak may show symmetrical properties both at room temperature and low temperatures. The SPR signal is in the form of Lorentzian and Gaussian line shapes at all temperature range. Specially prepared nanoparticles SPR peak exhibit shift in symmetry. The line width of SPR peak expands at low temperature.

\section{Author details}

Orhan Yalçın

Niğde University, Niğde, Turkey

\section{Acknowledgement}

I would like to thank Muhittin Öztürk and Songül Özüm of Niğde University for valuable discussions an the critical reading of the chapter. This study was supported by Research found (Grant No. FEB2012/12) of Niğde University.

\section{References}

Adeyeye, A.O.; Bland, J.A.C.; Daboo, C.; Hasko, D.G. (1997). Magnetostatic interactions and magnetization reversal in ferromagnetic wires. Phys. Rev. B. Vol. 56, pp. 3265.

Aktaş, B. (1993). Clear evidence for field induced unidirectional exchange surface anisotropy in NiMn alloys. Solid State Commun.Vol. 87, pp. 1067.

Aktaş, B.; Özdemir, M. (1994). Simulated spin wave resonance absorption curves for ferromagnetic thin films and application to NiMn films. Physica B. Vol. 119, pp. 125.

Aktaş, B. (1997). FMR properties of epitaxial $\mathrm{Fe}_{3} \mathrm{O}_{4}$ films on $\mathrm{MgO}(100)$. Thin Solid Films. Vol. 307, pp. 250.

Aktaş, B.; Özdemir, M.; Yilgin, R.; Öner, Y.; Sato, T.; Ando, T. (2001). Thickness and temperature dependence of magnetic anisotropies of Niz7 Mn23 films. Physica B. Vol. 305, pp. 298.

Aktaş, B.; Yildiz, F.; Rameev, B.; Khaibullin, R.; Tagirov, L.; Özdemir, M. (2004). Giant room temperature ferromagnetism in rutile $\mathrm{TiO}_{2}$ implanted by Co. Phys. stat. sol. (c). Vol. 12, pp. 3319.

Aktaş, B.; Tagirov, L. \& Mikailov, F. (October, 2006). Magnetic Nanostructures, Springer Series in materials science, Vol. 94, ISBN 978-3-540-49334-1. 
Ament, W. S.; Rado, G. T. (1955). Electromagnetic effects of spin wave resonance in ferromagnetic metals. Phys. Rev. Vol. 97, pp. 1558.

Ando, Y.; Koizumi, H.; Miyazaki, T. (1997). Exchange coupling energy determined by ferromagnetic resonance in $80 \mathrm{Ni}-\mathrm{Fe} / \mathrm{Cu}$ multilayer films. J. Magn. Magn. Mater. Vol. 166, pp. 75.

Anisimov, A. N.; Farle, M.; Poulopoulos, P.; Platow, W.; Baberschke, K.; Isberg, P.; Wäppling, R.; Niklasson, A. M. N.; Eriksson, O. (1999). Orbital magnetism and magnetic anisotropy probed with ferromagnetic resonance. Phys. Rev. Lett. Vol. 82, pp. 2390.

An, S. Y.; Krivosik, P.; Kraemer, M. A.; Olson, H. M.; Nazarov, A. V.; Patton, C. E. (2004). High power ferromagnetic resonance and spin wave instability processes in permalloy thin films. J. Appl. Phys. Vol. 96, pp. 1572.

Anderson, P. W. (1953). Exchange narrowing in paramagnetic resonance. Rev. Mod. Phy. Vol. 25, pp. 269.

Arias, R.; Mills, D. L. (1999). Extrinsic contributions to the ferromagnetic resonance response in ultrathin films, Phys. Rev. B. Vol. 60, pp. 7395.

Arias, R.; Mills, D. L. (2003). Theory of collective spin waves and microwave response of ferromagnetic nanowire arrays. Phys. Rev. B. Vol. 67, pp. 094423.

Arkad'ev, V.K. (1913). The Reflection of Electric Waves from a Wire, Sov. Phys.-JETP, Vol. 45A, issue 45, pp. 312.

Artman, J. O. (1957). Ferromagnetic resonance in metal single crystals. Phys. Rev. Vol. 105, pp. 74.

Artman, J. O. (1979). Domain mode FMR in materials with $K_{1}$ and $K_{u}$. J. Appl. Phys. Vol. 50, pp. 2024.

Astalos, R. J.; Camley, R. E. (1998). Magnetic permeability for exchange-spring magnets: application to Fe/Sm-Co. Phys. Rev. B. Vol. 58, pp. 8646.

Aswal, D.K.; Singh, A.; Kadam, R.M.; Bhide, M.K.; Page, A.G.; Bhattacharya, S.; Gupta, S.K.; Yakhmi, J.V.; Sahni, V.C. (2005). Ferromagnetic resonance studies of nanocrystalline La0.6 $\mathrm{Pb}_{0.4} \mathrm{MnO}_{3}$ thin films. Mater. Lett. Vol. 59, pp. 728.

Azzerboni, B.; Asti, G.; Pareti, L.; Ghidini, M. (2006). Magnetic nanostructures in modern technology, spintronics, magnetic MEMS and recording. Proceedings of the NATO advanced study institute on magnetic nanostructures for micro-electromechanical systems and spintronic applications catona, Published by Springer. Italy. ISBN 978-14020-6337-4.

Backes, D.; Bedau, D., Liu, H., Langer, J.; Kent, A.D. (2012). Characterization of interlayer interactions in magnetic random access memory layer stacks using ferromagnetic resonance. J. Appl. Phys. Vol. 111, pp. 07C721.

Baek, J.S.; Min, S.G.; Yu, S.C.; Lim, W.Y. (2002). Ferromagnetic resonance of Fe-Sm-O thin films. J. App. Phys. Vol. 93, pp. 7604.

Bai, Y .; Xu, F.; Qiao, L. (2012). The twice ferromagnetic resonance in hexagonal ferrite single rod and paired rods. Phys. Lett. A. Vol.376, pp. 563.

Bakker, F.L.; Flipse, J.; Slachter, A.; Wagenaar, D.; van Wees, B.J. (2012). Thermoelectric detection of ferromagnetic resonance of a nanoscale ferromagnet. Phys. Rev. Lett. Vol. 108, pp. 167602. 
Bakuzis, A.F.; Morais, P.C. (2004). Magnetic nanoparticle systems: an Ising model approximation. J. Magn. Magn. Mater. Vol. 272-276, pp. e1161.

Baseglia, L.; Warden, M.; Waldner, F.; Hutton, S.L.; Drumheller, J.E.; He, Y.Q.; Wigen, P.E.; Maryško M. (1988). Derivation of the resonance frequency from the free energy of ferromagnets. Phys. Rev. B. Vol. 38, pp. 2237.

Beguhn, S.; Zhou, Z.; Rand, S.; Yang, X.; Lou, J.; Sun, N.X. (2012). A new highly sensitive broadband ferromagnetic resonance measurement system with lock-in detection. $J$. Appl. Phys. Vol. 111, pp. 07A503.

Berger, R.; Bissey, J.; Kliava, J. (2000(a)). Lineshapes in magnetic resonance spectra. J. Phys.: Condens. Matter. Vol. 12, pp. 9347.

Berger, R.; Kliava, J.; Bissey, J.C. (2000(b)). Magnetic resonance of superparamagnetic ironcontaining nanoparticles in annealed glass. J. Appl. Phys. Vol. 87, pp. 7389.

Berger, R.; Bissey, J.C.; Kliava, J.; Soulard, B. (1997). Superparamagnetic resonance of ferric ions in devitrifield borate glass. J. Magn. Magn. Mater. Vol. 167, pp. 129.

Berger, R.; Bissey, J. C.; Kliava, J.; Daubric, H.; Estournès, C. (2001). Temperature dependence of superparamagnetic resonance of iron oxide nanoparticles. J. Magn. Magn. Mater. Vol. 234, pp. 535.

Berger, R.; Kliava, J.; Bissey, J. C.; Baïettoz, V. (1998). Superparamagnetic resonance of annealed iron containing borate glass. J. Phys.: Condens. Matter. Vol. 10, pp. 8559.

Birkhäuser Verlag, A.G. (2007). Spin glasses statics and dynamics. Basel, Boston, Berlin. ISBN 978-3-7643-8999-4.

Birlikseven, C.; Topacli, C.; Durusoy, H.Z.; Tagirov, L.R.; Koymen, A.R.; Aktaş, B. (1999 (a)). Magnetoresistance, magnetization and FMR study of Fe/Ag/Co multilayer film. J. Magn. Magn. Mater. Vol. 192, pp. 258.

Birlikseven, C.; Topacli, C.; Durusoy, H.Z.; Tagirov, L.R.; Koymen, A.R.; Aktaş, B. (1999 (b)). Layer-sensitive magnetization, magnetoresistance and ferromagnetic resonance (FMR) study of NiFe/Ag/CoNi trilayer film. J. Magn. Magn. Mater. Vol. 202, pp. 342.

Bhagat, S.M.; Anderson, J.R.; Wu, N. (1967). Influence of the anomalous skin effect on the ferromagnetic resonance linewidth in iron. Phys. Rev. Vol. 155, pp. 510.

Bhagat, S.M.; Lubitz, P. (1974). Temperature variation of ferromagnetic relaxation in the $3 d$ transition metals. Phys. Rev. B. Vol. 10, pp. 179.

Bland, J.A.C.; Heinrich, B. (1994). Ultrathin magnetic structures I: An introduction to the electronic magnetic and structural properties. Springer-Verlag Berlin Heidelberg. ISBN 3540-57407-7.

Bloembergen, B. (1950). On the ferromagnetic resonance in nickel and supermalloy. Phys. Rev. Vol. 78, pp. 572.

Bloembergen, N.; Wang, S. (1954). Relaxation effects in para- and ferromagnetic resonace. Phys. Rev. Vol. 93, pp. 72.

Borchers, J.A.; Dura, J.A.; Unguris, J.; Tulchinsky, D.; Kelley, M.H.; Majkrzak, C.F. (1998). Observation of antiparallel magnetic order in weakly coupled CoyCu multilayers. Phys. Rev. Lett. Vol. 82, pp. 2796.

Bose, T.; Trimper, S. (2012). Nonlocal feedback in ferromagnetic resonance. Arxiv: 1204-5342. Cond-mat. Mes-hall. pp. 1. 
Boulle, O.; Malinowski, G.; Kläui, M. (2011). Current-induced domain wall motion in nanoscale ferromagnetic elements. Materials Science and Engineering R. Vol. 72, pp. 159.

Brown, F.M. (1962).Magnetostatic principles in ferromagnetism. North Holland Publishing Company.

Brustolon, M.; Giamello, E. (2009). Electron paramagnetic resonance. John Wiley E Sons, Inc. ISBN 978-0-470-25882.

Budak, S.; Yildiz, F.; Özdemir, M.; Aktaş, B. (2003). Electron spin resonance studies on single crystalline $\mathrm{Fe}_{3} \mathrm{O}_{4}$ films. J. Magn. Magn. Mater. Vol. 258-259, pp. 423.

Buschow, K.H.J.; de Boer, F.R. (2004). Physics of magnetism and magnetic materials. Kluwer Academic Publishers. ISBN: 0-306-47421-2.

Callen, H.B. (1958). A ferromagnetic dynamical equation. J. Phys. Chem. Solids. Vol. 4, pp. 256.

Can, M.M.; Coşkun, M.; Fırat, T. (2012). A comparative study of nanosized iron oxide particles; magnetite $\left(\mathrm{Fe}_{3} \mathrm{O}_{4}\right)$, maghemite $\left(\gamma-\mathrm{Fe}_{2} \mathrm{O}_{3}\right)$ and hematite $\left(\alpha-\mathrm{Fe}_{2} \mathrm{O}_{3}\right)$, using ferromagnetic resonance. J. Alloy. Compd. Vol. 542, pp. 241.

Celinski, Z.; Urquhart, B.; Heinrich, B. (1997). Using ferromagmetic resonance to measure the magnetic moments of ultrathin films, J. Magn. Magn. Mater. Vol. 166, pp. 6.

Celinski, Z.; Heinrich, B. (1991). Ferromagnetic resonance line width of Fe ultrathin films grown on a bcc substrate. J. Appl. Phys. Vol. 70, pp.5936.

Chappert, C.; Le Dang, K.; Beauvillain, P.; Hurdequint, H.; Renard, D. (1986). Ferromagnetic resonance studies of very thin cobalt films on a gold substrate. Phys. Rev. B. Vol. 34, pp. 3192.

Chastellain, M.; Petri, A.; Hofmann, H. (2004). Particle size investigations of a multi-step synthesis of PVA coated superparamagnetic nanoparticles. J. Colloid. Interf. Sci. Vol. 278, pp. 353.

Chen, Y.S.; Cheng, C.W.; Chern, G.; Wu, W.F.; Lin, J.G. (2012). Ferromagnetic resonance probed annealing effects on magnetic anisotropy of perpendicular $\mathrm{CoFeB} / \mathrm{MgO}$ bilayer. J. Appl. Phys. Vol. 111, pp.07C101.

Chen, S.H.; Chang, C.R.; Xiao, J.Q.; Nikolić, K.B. (2009). Spin and charge pumping in magnetic tunnel junctions with precessing magnetization: A nonequilibrium green function approach. Phys. Rev. B. Vol. 79, pp. 054424.

Chen, H.H.; Levy, P.M. (1973). Dipole and quadrupole phase transitions in spin-1 models. Phys. Rev. B. Vol. 7, 4267.

Chikazumi, S. (1997). Physics of ferromagnetism. Oxford University Press. ISBN 0-19-8517769.

Cochran, J.F. (1995). Light scattering from ultrathin magnetic layers and bilayers in magnetic ultrathin films. Heinrich, B.; Bland, J.A.C. (Eds.) Springer, Berlin, Heidelberg Vol. II, pp. 222. B. Hillebrands: Brillouin light scattering in magnetic superlattices. ibid pp. 258.

Cochran, J.F.; Kambersky, V. (2006). Ferromagnetic resonance in very thin films. J. Magn. Magn. Mater. Vol. 302, pp. 348.

Coey, J.M.D. (1997). Permanent magnetism. Solid State Comm. Vol. 102, pp. 101.

Coey, J.M.D. (2009). Magnetism and magnetic materials. Cambridge University Press. ISBN-13 978052181614-4. 
Cofield, M.L.; Glocker, D.; Gau, J.S. (1987). Spin-wave resonance in CoCr magnetic thin films. J. Appl. Phys. Vol. 61, pp. 3810.

Coşkun, R.; Okutan, M.; Yalçın, O.; Kösemen, A. (2012). Electric and magnetic properties of hydrogels doped with Cu ions. Acta. Phys. Pol. A. Vol. 122, pp. 683.

Crittenden, E.C.Jr; Hoffman, R.W. (1953). Thin films of ferromagnetic materials. Rev. Mod. Phys. Vol. 25, pp. 310.

Cullity, B.D.; Graham, C.D. (1990). Introduction to Magnetic Materials. Wiley. New York. pp. 199.

Cullity, B.D.; Graham, C.D. (2009). Introduction to magnetic materials $2^{\text {nd }}$ Edition.

Damon, R.W. (1953). Relaxation effect in the ferromagnetic resonance. Rev. Mod. Phys. Vol. 25 , pp. 239.

Damon, R.W. (1963). Ferromagneticre sonance at high power in Rado, G.T.; Suhl, H. (Eds.). Magnetism. Vol. I

da Silva, E.C.; Meckenstock, R.; von Geisau, O.; Kordecki, R.; Pelzl, J.; Wolf, J.A.; Grünberg, P. (1993). Ferromagnetic resonance investigations of anisotropy fields of $\mathrm{Fe}(001)$ epitaxial layers. J. Magn. Magn. Mater. Vol. 121, pp. 528.

Davis, J.A. (1965). Effect of surface pinning on the magnetization of thin films. J. Appl. Phys. Vol. 36, pp. 3520.

Demokritov, S.; Rücker, U.; Grünberg, P. (1996). Enhancement of the Curie temperature of epitaxial EuS(100) films caused by growth dislocations. J. Magn. Magn. Mater. Vol. 163, pp. 21.

Demokritov, S.; Rücker, U.; Arons, R.R.; Grünberg, P. (1997). Antiferromagnetic interlayer coupling in epitaxial Fe/EuS (100) bilayers. J. Appl. Phys. Vol. 81, pp. 5348.

De Wames, R.E.; Wolfram, T. (1970). Dipole-exchange spinwaves in ferromagnetic films. J. Appl. Phys. Vol. 41, pp. 87.

Demand, M.; Encinas-Oropesa, A.; Kenane, S.; Ebels, U.; Huynen, I.; Pirax, L. (2002). Ferromagnetic resonance studies of nickel and permalloy nanowire arrays. J. Magn. Magn. Mater.Vol. 249, pp. 228.

De Biasi, E.; Lima, Jr. E.; Ramos, C.A.; Butera, A.; Zysler, R.D. (2013). Effect of thermal fluctuations in FMR experiments in uniaxial magnetic nanoparticles: Blocked vs. superparamagnetic regimes. J. Magn. Magn. Mater. Vol. 326, pp. 138.

De Biasi, R.S.; Gondim, E.C. (2006). Use of ferromagnetic resonance to determine the size distribution of $\gamma-\mathrm{Fe}_{2} \mathrm{O}_{3}$ nanoparticles. Solid State Commun. Vol. 138, pp. 271.

De Cos, D.; Arribas, A.G.; Barandiaran, J.M. (2006). Ferromagnetic resonance in gigahertz magneto-impedance of multilayer systems. J. Magn. Magn. Mater. Vol. 304, pp. 218.

Diaz, L.L.; Torres, L.; Moro, E. (2002). Transition from ferromagnetism to superparamagnetism on the nanosecond time scale. Phys. Rev. B. Vol. 65, pp. 224406.

Dillon, J.F.Jr.; Gyorgy, E.M.; Rupp, L.W.Jr.; Yafet, Y.; Testardi,L.R. (1981). Ferromagnetic resonance in compositionally modulated CuNi films. J. Appl. Phys. Vol. 52, pp. 2256.

Ding, J.; Kostylev, M.; Adeyeye, A.O. (2012). Broadband ferromagnetic resonance spectroscopy of permalloy triangular nanorings. J. Appl. Phys. Vol. 100, pp. 062401. 
Dormer, K.; Seeney, C.; Lewelling, K.; Lian, G.; Gibson, D.; Johnson, M. (2005). Epithelial internalization of superparamagnetic nanoparticles and response to external magnetic field. Biomaterials. Vol. 26, pp. 2061.

Dreher, L.; Weiler, M.; Pernpeintner, M.; Huebl, H.; Gross, R.; Brandt, M.S.; Goennenwein, S.T.B. (2012). Surface acoustic wave-driven ferromagnetic resonance in nickel thin films: Theory and experiment. Arxiv:Cond-mat. Mes-hall. Vol. 1208, pp. 1.

Dubowik, J. (1996). Shape anisotropy of magnetic heterostructures. Phys. Rev. B. Vol. 54, pp.1088.

Dumesnil, K.; Dufour, C.; Mangin, Ph.; Rogalev, A. (2002). Magnetic springs in exchangecoupled $\mathrm{D}_{\mathrm{y}} \mathrm{Fe}_{2} / \mathrm{YFe}_{2}$ superlattices: An element-selective $\mathrm{x}$-ray magnetic circular dichroism study. Phys. Rev. B. Vol. 65, pp. 094401.

Duraia, E.M.; Abdullin, Kh.A. (2009). Ferromagnetic resonance of cobalt nanoparticles used as a catalyst for the carbon nanotubes synthesis. J. Magn. Magn. Mater. Vol. 321, pp. 69.

Durusoy, H.Z.; Aktaş, B.; Yilgin, R.; Terada, N.; Ichikawa, M.; Kaneda, T.; Tagirov, L.R. (2000). New technique for measuring the microwave penetration depth in high- $T_{c}$ superconducting thin films. Phys. B. Vol. 284-288, pp. 953.

Encinas-Oropesa, A.; Demand, M.; Piraux, L.; Huynen, I.; Ebels, U. (2001). Dipolar interactions in arrays of nickel nanowires studied by ferromagnetic resonance. Phys. Rev. B. Vol. 63, pp. 104415.

Erdem, R.; Keskin, M. (2001). Dynamics of a spin-1 Ising system in the neighborhood of equilibrium states, Phys. Rev. E. Vol. 64, pp. 026102.

Erkovan, M.; Öztürk, S.T.; Topkaya, R.; Özdemir, M.; Aktaş, B.; Öztürk, O. (2011). Ferromagnetic resonance investigation of $\mathrm{Py} / \mathrm{Cr}$ multilayer system. J. Appl. Phys. Vol. 110, pp. 023908.

Estévez, D.C.; Betancourt, I.; Montiel, H. (2012). Magnetization dynamics and ferromagnetic resonance behavior of melt spun FeBSiGe amorphous alloys. J. Appl. Phys. Vol. 112, pp. 053923.

Fan, W.J.; Qiu, X.P.; Shi, Z.; Zhou, S.M.; Cheng, Z.H. (2010). Correlation between isotropic ferromagnetic resonance field shift and rotatable anisotropy in polycrystalline NiFe/FeMn bilayers. Thin Solid Films. Vol. 518, pp. 2175.

Farle, M. (1998). Ferromagnetic resonance of ultrathin metallic layers. Rep. Prog. Phys. Vol. 61, pp. 755.

Farle, M.; Lindner, J.; Baberschke, K. (2000). Ferromagnetic resonance of Ni(111) on $\operatorname{Re}(0001)$. J. Magn. Magn. Mater. Vol. 212, pp. 301.

Fermin, J.R.; Azevedo, A.; Aguiar, F.M.; Li, B.; Rezende, S.M. (1999). Ferromagnetic resonance linewidth and anisotropy dispersion in thin Fe films. J. Appl. Phys. Vol. 85, pp. 7316.

Fischer, H.; Mastrogiacomo, G.; Löffler, J.F.; Warthmann, R.J.; Weidler, P.G.; Gehring, A.U. (2008). Ferromagnetic resonance and magnetic characteristics of intact magnetosome chains in Magnetospirillum gryphiswaldense. Earth and Planetary Science Letters. Vol. 270, pp. 200.

Fletcher, R.C.; Le Craw, R.C.; Spencer, E.G. (1960). Electron spin relaxation in ferromagnetic insulators. Phys. Rev. Vol. 117, pp. 955. 
Fonseca, F.C.; Goya, G.F.; Jardim, R.F.; Muccillo, R.; Carreño, N.L.V.; Longo, E.; Leite, E.R. (2002). Superparamagnetism and magnetic properties of Ni nanoparticles embedded in SiO2. Phys. Rev. B. Vol. 66, pp.104406.

Frait, Z.; Macfaden, H. (1965). Ferromagnetic resonance in metals frequency dependence. Phys. Rev. Vol.139, pp.A1173.

Frait, Z.; Fraitova, D.; Zarubova, N. (1985). Observation of FMR surface spin wave modes in bulk amorphous ferromagnets. Phys. Stat. Sol. (b). Vol. 128, pp. 219.

Frait, Z.; Fraitova, D. (1998). Low energy spinwave excitation in highly conducting thin films and surfaces, in Frontiers in Magnetism of Reduced Dimension Systems. Nato ASI Series, Bar'yakhtar, V.G.; Wigen, P.E.; Lesnik, N.A. (Eds.) (Kluwer, Dordrecht) pp. 121.

Frait, Z.; Macfaden, H. (1965). Ferromagnetic resonance in metals frequency dependence. Phys. Rev. Vol. 139, pp. 1173.

Fraitova, D. (1983(a)). An analytical theory of FMR in bulk metals, I. Dispersion relations. Phys. Stat. Sol. Vol. 120, pp. 341.

Fraitova, D. (1983(b)). An analytical theory of FMR in bulk metals, II. Penetration depths. Phys. Stat. Sol. Vol. 120, pp. 659.

Fraitova, D. (1984). An analytical theory of FMR in bulk metals, III. Surface impedance. Phys. Stat. Sol. Vol. 124, pp. 587.

Fullerton, E.E.; Jiang, J.S.; Bader, S.D. (1999). Hard/soft magnetic heterostructures: model exchange-spring magnets. J. Magn. Magn. Mater. Vol. 200, pp. 392.

Fullerton, E.E.; Jiang, J.S.; Grimsditch, M.; Sowers, C.H.; Bader, S.D. (1998). Exchange-spring behavior in epitaxial hard/soft magnetic bilayers. Phys. Rev. B. Vol. 58, pp.12193.

Gatteschi, D.; Sessoli, R.; Villain, J. (2006). Molecular nanomagnets. Oxford University Press.

García-Miquel, H.; García, J.M.; García-Beneytez, J.M.; Vázquez, M. (2001). Surface magnetic anisotropy in glass-coated amorphous microwires as determined from ferromagnetic resonance measurements. J. Magn. Magn. Mater. Vol. 231, pp. 38.

Gerlach, W.; Stern, O. (1922). Dasmagnetische moment dessilber atoms. Zeitschrift für Physik. Vol. 9, pp. 353.

Gilbert, T.A. (1955). Armour research foundation rept. Armour Research Foundation. Chicago. No.11.

Gnatzig, K.;. Dötsch, H.; Ye, M.; Brockmeyer, A. (1987). Ferrimagnetic resonance in garnet films at large precession angles. J. Appl. Phys. Vol. 62, pp. 4839. Golosovsky, M.; Monod, P.; Muduli, P.K.; Budhani, R.C. (2012). Low-field microwave absorption in epitaxial La0.7Sro. $\mathrm{MnO}_{3}$ films resulting from the angle-tuned ferromagnetic resonance in the multidomain state. Arxiv: 1206-3041. Cont-mat. mtrl-sci. pp. 1.

Goryunov, Yu. V.; Garifyanov, N.N.; Khaliullin, G.G.; Garifullin, I.A.; Tagirov, L.R.; Schreiber, F.; Mühge, Th.; Zabel, H. (1995). Magnetican isotropies of sputtered Fe films on MgO substrates. Phys. Rev. B. Vol. 52, pp. 13450.

Grimsditch, M.; Camley, R.; Fullerton, E.E.; Jiang, S.; Bader, S.D.; Sowers, H. (1999). Exchange-spring systems: Coupling of hard and soft ferromagnets as measured by magnetization and Brillouin light scattering (invited). J. Appl. Phys. Vol. 85, pp. 5901.

Grünberg, P.; Schwarz, B.; Vach, W.; Zinn, W.; Dabkowski, D. (1979). Light scattering from spin waves in bubble films. J. Magn. Magn. Mater. Vol. 13, pp. 181. 
Grünberg, P. (1980). Brillouin scattering from spin waves in thin ferromagnetic films. J. Magn. Magn. Mater. Vol. 15-18, pp. 766.

Grünberg, P.; Mayr, C.M.; Vach, W. (1982). Determination of magnetic parameters by means of brillouin scattering. Examples: Fe, Ni, Nio.seo.2. J. Magn. Magn. Mater. Vol. 28, pp. 319.

Grünberg, P.; Barnas, J.; Saurenbach, F.; Fuß, J.A.; Wolf, A.; Vohl, M. (1991). Layered magnetic structures: antiferromagnetic type interlayer coupling and magnetoresistance due to antiparallel alignment. J. Magn. Magn. Mater. Vol. 93, pp. 58.

Grünberg, P.; Demokritov, S.; Fuss, A.; Schreiber, R.; Wolf, J.A.; Purcell, S.T. (1992). Interlayer exchange, magnetotransport and magnetic domains in $\mathrm{Fe} / \mathrm{Cr}$ layered structures. J. Magn. Magn. Mater. Vol. 104-107, pp. 1734.

Grünberg, P. (2000). Layered magnetic structures in research and application. Acta mater. Vol. 48, pp. 239. Grünberg, P. (2001). Layered magnetic structures: history, facts and figures. J. Magn. Magn. Mater. Vol. 226-230, pp. 1688.

Guimarães, A.P. (2009). Principles of nanomagnetism. Springer-Verlag Berlin Heidelberg. ISBN 978-3-642-01481-9. Guimarães, A.P. (1998). Magnetism and magnetıc resonance in solids. A Wiley-Interscience Publication. Canada.

Gurevich, A.G.; Melkov, G.A. (1996). Magnetization Oscillations and Waves (CRC, Boca Raton).

Güner, S.; Yalçın, O.; Kazan, S.; Yıldız, F.; Şahingöz, R. (2006). FMR studies of bilayer $\mathrm{C}_{90} \mathrm{Fe}_{10} / \mathrm{Ni}_{81} \mathrm{Fe}_{19}, \mathrm{Ni}_{18} \mathrm{Fe}_{19} / \mathrm{Co}_{0} \mathrm{Fe}_{10}$ and monolayer Nis1 Fe19 thin films. Phys. Stat. Solid (a). Vol. 203, pp. 1539.

Hamoudeh, M.; Al Faraj, A.; Canet-Soulas, E.; Bessueille, F.; Leonard, D.; Fessi, H. (2007). Elaboration of PLLA-based superparamagnetic nanoparticles: Characterization, magnetic behaviour study and in vitro elaxivity evaluation. Int. J. Pharmaceut. Vol. 338, pp. 248.

Hathaway, K.; Cullen, J. (1981). Magnetoelastic softening of moduli and determination of magnetic anisotropy in RE-TM compounds. J. Appl. Phys. Vol. 52, pp. 2282.

Heim, E.; Harling, S.; Pöhlig, K.; Ludwig, F.; Menzel, H.; Schilling, M. (2007). Fluxgate magnetorelaxometry of superparamagnetic nanoparticles for hydrogel characterization. J. Magn. Magn. Mater. Vol. 311, pp. 150.

Heinrich, B. (2005(a)). Ferromagnetic resonance in ultrathin film structures. In magnetic ultrathin films. Heinrich, B.; Bland, J.A.C. (Eds.) Springer, Berlin, Heidelberg. Vol. II, pp. 195.

Heinrich, B.; Bland, J.A.C. (2005(b)). Ultrathin magnetic structures IV, applications of nanomagnetism. Springer Berlin Heidelberg New York . ISBN 3-540-21954-4.

Heisenberg, W. (1928). Theory of ferromagnetism. ZeitschriftfürPhysik. Vol. 49, pp. 619.

Held, G.A.; Grinstein, G.; Doyle, H.; Sun, S.; Murray, C.B. (2001). Competing interactions in dispersions of superparamagnetic nanoparticles. Phys. Rev. B. Vol. 64, pp. 012408.

Hellwig, O.; Kortrigh, J.B.; Takano, K.; Fullerton, E.E. (2000). Switching behavior of Fe-Pt/NiFe exchange-

spring films studied by resonant soft-x-ray magneto-optical Kerr effect. Phys. Rev. B. Vol. 62, pp. 11694. 
Herring, C.; Kittel, C. (1950). On the theory of spin waves in ferromagnetic media. Phys. Rev. Vol. 81, pp. 869.

Hillebrands, B. (2000). Light scattering in solids VII. Topics. Appl. Phys. Vol. 75, M. Cardona, Güntherodt, G. (Eds.). Springer, Berlin, Heidelberg.

Hillebrands, B.; Thiaville, A. (2006). Spin dynamics in confined magnetic structures III. Springer-Verlag Berlin Heidelberg. ISBN-10 3-540-20108-4.

Hillebrands, B.; Ounadjela, K. (2002). Spin dynamics in confined magnetic structures I. Springer-Verlag Berlin Heidelberg.

Hillebrands, B.; Ounadjela, K. (2003). Spin dynamics in confined magnetic structures II. Springer-Verlag Berlin Heidelberg. Hinata, S.; Saito, S.; Takahashi, M. (2012). Ferromagnetic resonance analysis of internal effective field of classified grains by switching field for granular perpendicular recording media. J. Appl. Phys.. Vol. 111, pp. $07 \mathrm{~B} 722$.

Hsia, L.C.; Wigen, P.E. (1981). Enhancement of uniaxial anisotropy constant by introducing oxygen vacancies in Ca-doped YIG. J. Appl. Phys. Vol. 52, pp. 1261.

Huang, Z.C.; Hu, X.F.; Xu, Y.X.; Zhai, Y.; Xu, Y.B.; Wu, J.; Zhai, H. R. (2012). Magnetic properties of ultrathin single crystal $\mathrm{Fe}_{3} \mathrm{O}_{4}$ film on $\mathrm{InAs}(100)$ by ferromagnetic resonance. J. Appl. Phys. Vol. 111, pp. 07C108.

Huang, M.D.; Lee, N.N.; Hyun, Y.H.; Dubowik, J.; Lee, Y.P. (2004). Ferromagnetic resonance study of magnetic-shape-memory $\mathrm{Ni}_{2} \mathrm{MnGa}$ films. J. Magn. Magn. Mater. Vol. 272-276, pp. 2031.

Jalali-Roudsar, A.A.; Denysenkov, V.P.; Khartsev, S.I. (2005). Determination of magnetic anisotropy constants for magnetic garnet epitaxial films using ferromagnetic resonance. J. Magn. Magn. Mater. Vol. 288, pp. 15.

Jarrett, H.S.; Waring, R.K. (1958). Ferrimagnetic resonance in $\mathrm{NiMnO}_{3}$. Phys. Rev. Vol. 111, pp. 1223.

Jiang, J.S.; Fullerton, E.E.; Sowers, C.H.; Inomata, A.; Bader, S.D.; Shapiro, A.J.; Shull, R.D.; Gornakov, V. S.; Nikitenko, V.I. (1999). Spring magnet films. IEEE Trans. Magn. Vol. 35, pp. 3229.

Jiang, J.S.; Pearson, J.E.; Liu, Z.Y.; Kabius, B.; Trasobares, S.; Miller, D.J.; Bader, S.D. (2005). A new approach for improving exchange-spring magnets. J. Appl. Phys. Vol. 97, pp. 10K311.

Jiang, J.S.; Bader, S.D.; Kaper, H.; Leaf, G.K.; Shull, R.D.; Shapiro, A.J.; Gornakov, V.S.; Nikitenko, V.I.; Platt, C.L.; Berkowitz, A.E.; David, S.; Fullerton, E.E. (2002). Rotational hysteresis of exchange-spring Magnets. J. Phys. D: Appl. Phys. Vol. 35, pp. 2339.

Jung, S.; Watkins, B.; DeLong, L.; Ketterson, J.B.; Chandrasekhar, V. (2002). Ferromagnetic resonance in periodic particle arrays. Phys. Rev. B. Vol. 66, pp. 132401.

Kakazei, G.N.; Kravets, A.F.; Lesnik, N.A.; de Azevedo, M.M.P.; Pogorelov, Y. G.; Sousa, J.B. (1999). Ferromagnetic resonance in granular thin films. J. Appl. Phys. Vol. 85, pp. 5654.

Kakazei, G.N.; Pogorelov, Yu.G.; Sousa, J.B.; Golub, V.O.; Lesnik, N.A.; Cardoso, S.; Freitas, P.P. (2001). FMR in $\mathrm{CoFe}(t) / \mathrm{Al}_{2} \mathrm{O}_{3}$ multilayers: from continuous to discontinuous regime. J. Magn. Magn. Mater. Vol. 226-230, pp. 1828. 
Kambe, T.; Kajiyoshi, K.; Oshima, K.; Tamura, M.; Kinoshita, M. (2005). Ferromagnetic resonance in $\beta$-p-NPNN at radio-frequency region. Polyhedron. Vol. 24, pp. 2468.

Kartopu, G.; Yalçın, O.; Kazan, S.; Aktaş, B. (2009). Preparation and FMR analysis of Co nanowires in alumina templates. J. Magn. Magn. Mater. Vol. 321, pp. 1142.

Kartopu, G.; Yalçın, O. (2010). Electrodeposited nanowires and their applications. edited by N. Lupu. INTECH. available from: http://sciyo.com/articles/show/title/fabrication-andapplications-of-metalnanowire-arrays-electrodeposited-in-ordered-porous-templates.

Kartopu, G.; Yalçın, O.; Choy, K.-L.; Topkaya, R.; Kazan, S.; Aktaş, B. (2011 (a)). Size effects and origin of easy-axis in nickel nanowire arrays. J. Appl. Phys. Vol. 109, pp. 033909.

Kartopu, G; Yalçın, O; Demiray, A.S. (2011 (b)). Magnetic and transport properties of chemical solution deposited (100)-textured La0.7 $\mathrm{Sr}_{0.3} \mathrm{MnO}_{3}$ and $\mathrm{La}_{0 .} \mathrm{Ca}_{0.3} \mathrm{MnO}_{3}$ nanocrystalline thin films. Phys. Scr. Vol. 83, pp. 015701.

Kasuya, T. (1956). A theory of metallic ferro- and antiferromagnetism on Zener's model. Prog. Theor. Phys. Vol. 16, pp. 45.

Kind, J.; van Raden, U.J.; García-Rubio, I.; Gehring, A.U. (2012). Rock magnetic techniques complemented by ferromagnetic resonance spectroscopy to analyse a sediment record. Geophys. J. Int. Vol. 191, pp. 51.

Kip, A.F.; Arnold, R.D. (1949). Ferromagnetic resonance at microwave frequencies in iron single crystal. Phys. Rev. Vol. 75, pp. 1556.

Kittel, C. (1947). On the theory of ferromagnetic resonance absorption. Phys. Rev. Vol. 73, pp. 155.

Kittel, C. (1946).Theory of the structure of ferromagnetic domains in films and small Particles. Phys. Rev. Vol. 70, pp. 965.

Kittel, C. (1949). On the gyromagnetic ratio and spectroscopic splitting factor of ferromagnetic substances. Phys. Rev. Vol. 76, pp. 743.

Kittel, C.; Abrahams, E. (1953). Relaxation processes in ferromagnetism. Rev. Mod. Phys. Vol. 25, pp. 233.

Kip, A.F.; Arnold, R.D. (1949). Ferromagnetic Resonance at Microwave Frequencies in Iron Single Crystal. Phys. Rev. Vol. 75, pp. 1556.

Kittel, C. (1958). Interaction of spin waves and ultrasonic waves in ferromagnetic crystals. Phys. Rev. Vol. 110, pp. 836.

Kharmouche, A.; Ben Youssef, J.; Layadi, A.; Chérif, S.M. (2007). Ferromagnetic resonance in evaporated $\mathrm{Co} / \mathrm{Si}(100)$ and Co/glass thin films. J. App. Phys. Vol. 101, pp. 13910.

Khaibullin, R.I.; Tagirov, L.R.; Rameev, B.Z.; Ibragimov, S.Z.; Yıldız, F.; Aktaş, B. (2004). High curie-temperature ferromagnetism in cobalt-implanted single-crystalline rutile. $J$. Phys.: Condens. Matter. Vol. 16, pp. 1.

Klein, P.; Varga, R.; Infante, G.; Vázquez, M. (2012). Ferromagnetic resonance study of FeCoMoB microwires during devitrification process. J. Appl. Phys. Vol. 111, pp. 053920.

Kliava, J.; Berger, R. (1999). Size and shape distribution of magnetic nanoparticles in disordered systems: computer simulations of superparamagnetic resonance spectra. $J$. Magn. Magn. Mater. Vol. 205, pp. 328.

Kneller, E.F.; Hawing, R. (1991). The exchange-spring magnet: a new material principle for permanent magnets. IEEE Trans. Magn. Vol. 27, pp. 3588. 
Kobayashi, T.; Ishida, N.; Sekiguchi, K.; Nozaki, Y. (2012). Ferromagnetic resonance properties of granular $\mathrm{Co}-\mathrm{Cr}-\mathrm{Pt}$ films measured by micro-fabricated coplanar waveguides. J. Appl. Phys. Vol. 111, pp. 07B919.

Kodama, R.H. (1999). Magnetic Nanoparticles. J. Magn. Magn. Mater. Vol. 200, pp. 359.

Kodama, R.H.; Berkowitz, A.E. (1999). Atomic-scale magnetic modeling of oxide nanoparticles. Phys. Rev. B. Vol. 59, pp. 6321.

Kodama, R.H.; Berkowitz, A.E.; McNiff Jr. E.J.; Foner S. (1996). Surface spin disorder in $\mathrm{NiFe}_{2} \mathrm{O}_{4}$ nanoparticles. Phys. Rev. Lett. Vol. 77, pp. 394.

Kooi, C.F.; Wigen, P.E.; Shanabarger, M.R.; Kerrigan J.V. (1964). Spin-wave resonance in magnetic films on the basis of surface -spin -pinning model and the volume inhomogeneity model. J. Appl. Phys. Vol. 35, pp. 791.

Kopp, R.E.; Weiss, B.P.; Maloof, A.C.; Vali, H.; Nash, C.Z.; Kirschvink, J.L. (2006). Chains, clumps, and strings: magneto fossil taphonomy with ferromagnetic resonance spectroscopy. Earth Planet Sc. Lett. Vol. 247, pp. 10.

Korolev, K.A.; McCloy, J.S.; Afsar, M.N. (2012). Ferromagnetic resonance of micro- and nano-sized hexagonal ferrite powders at millimeter waves. J. Appl. Phys. Vol. 111, pp. $07 \mathrm{E} 113$.

Kohmoto, O. (2007). Ferromagnetic resonance equation of hexagonal ferrite in c-plane. J. Magn. Magn. Mater. Vol. 310, pp. 2561.

Knorr, T.G.; Hoffman, R.W. (1959). Dependence of geometric magnetic anisotropy in thin iron films. Phys. Rev. Vol. 113, pp. 1039.

Kraus, L.; Frait, Z.; Ababei, G.; Chayka, O.; Chiriac, H. (2012). Ferromagnetic resonance in submicron amorphous wires. J. Appl. Phys. Vol. 111, pp. 053924.

Krebs, J.J.; Rachford, F.J.; Lubitz, P.; Prinz, G.A. (1982). Ferromagnetic resonance studies of very thin epitaxial single crystals of iron. J. Appl. Phys. Vol. 53, pp. 8058.

Krebs, J.J.; Lubitz, P.; Chaiken, A.; Prinz, G.A. (1989). Magnetic resonance determination of the antiferromagnetic coupling of Fe layers through Cr. Phys. Rev. Lett. Vol. 63, pp. 1645.

Krivoruchko, V.N.; Marchenko, A.I. (2012). Spatial confinement of ferromagnetic resonances in honeycomb antidot lattices. J. Magn. Magn. Mater. Vol. 324, pp. 3087.

Krone, P.; Albrecht, M.; Schrefl, T. (2011). Micromagnetic simulation of ferromagnetic resonance of perpendicular granular media: Influence of the intergranular exchange on the Landau-Lifshitz-Gilbert damping constant. J. Magn. Magn. Mater. Vol. 323, pp. 432.

Kuanr, B.K.; Camley, R.E.; Celinski, Z. (2004). Relaxation in epitaxial Fe films measured by ferromagnetic resonance. J. Appl. Phys. Vol. 93, pp. 6610.

Kuanr, B.K.; Camley, R.E.; Celinski, Z. (2005). Extrinsic contribution to Gilbert damping in sputtered NiFe films by ferromagnetic resonance. J. Magn. Magn. Mater. Vol. 286, pp. 276.

Kudryavtsev, Y.V.; Oksenenko, V.A.; Kulagin, V.A.; Dubowik, J.; Lee, Y.P. (2007). Ferromagnetic resonance in Co2MnGa films with various structural ordering. J. Magn. Magn. Mater. Vol. 310, pp. 2271.

Lacheisserie, E.T.; Gignoux, D.; Schlenker, M. (2005). Magnetism, materials and aplications. Springer science + Business Media, Inc. Boston. 
Landau, E.; Lifshitz, E. (1935). On the theory of the dispersion of magnetic permeability in ferromagnetic bodies. Physik Z. Sowjetunnion. Vol. 8, pp. 153. Layadi, A.; Lee, J.M.; Artman, J.O. (1988). Spin-wave FMR in annealed NiFe/FeMn thin films. J. Appl. Phys. Vol. 63, pp. 3808.

Layadi, A. (2002). Exchange anisotropy: A ferromagnetic resonance study. Phys. Rev. B. Vol. 66, pp. 184423.

Layadi, A. (2004). Theoretical study of resonance modes of coupled thin films in the rigid layer model. Phys. Rev. B. Vol. 69, pp. 144431.

Layadi, A.; Artman, J.O. (1990(a)). Ferromagnetic resonance in a coupled two-layer System. J. Magn. Magn. Mater. Vol. 92, pp. 143.

Layadi A.; Artman, J.O. (1990(b)). Study of antiferromagnetic coupling by ferromagnetic resonance (FMR).J. Magn. Magn. Mater. Vol. 92, pp. 143.

Lee, J.; Hong, Y.K.; Lee, W.; Abo, G.S.; Park, J.; Syslo, R.; Seong, W.M.; Park, S.H.; Ahn, W.K. (2012). High ferromagnetic resonance and thermal stability spinel Nio.7Mn0.3-x $\mathrm{Co}_{x} \mathrm{Fe}_{2} \mathrm{O}_{4}$ ferrite for ultra high frequency devices. J. Appl. Phys. Vol. 111, pp. 07A516.

Limmer, W.; Glunk, M.; Daeubler, J.; Hummel, T.; Schoch, W.; Bihler, C.; Huebl, H.; Brandt, M.S.; Goennenwein, S.T.B.; Sauer, R. (2006). Magnetic anisotropy in (Ga, Mn)As on $\operatorname{GaAs}(1 \quad 13)$ As studied by magnetotransport and ferromagnetic resonance. Microelectron. J. Vol. 37, pp. 1490.

Lindner, J.; Baberschke, K. (2003). In situ ferromagnetic resonance: an ultimate tool to investigate the coupling in ultrathin magnetic films. J. Phys.: Condens. Matter. Vol. 15, pp. R193.

Lindner, J.; Tolinski, T.; Lenz, K.; Kosubek, E.; Wende, H.; Baberschke, K.; Ney, A.; Hesjedal, T.; Pampuch, C.; Koch, R.; Däweritz, L.; Ploog, K.H. (2004). Magnetic anisotropy of MnAs-films on GaAs(0 0 1) studied with ferromagnetic resonance. J. Magn. Magn. Mater. Vol. 277, pp. 159.

Li, N.; Schäfer, S.; Datta, R.; Mewes, T.; Klein, T. M.; Gupta, A. (2012). Microstructural and ferromagnetic resonance properties of epitaxial nickel ferrite films grown by chemical vapor deposition. Appl. Phys. Lett. Vol. 101, pp. 132409.

Liua, B.; Yang, Y.; Tang, D.; Zhang, B.; Lu, M.; Lu, H. (2012). The contributions of intrinsic damping and two magnon scattering on the ferromagnetic resonance linewidth in $\left[\mathrm{Fe}_{65} \mathrm{CO}_{35} / \mathrm{SiO}_{2}\right]_{\mathrm{n}}$ multilayer films. J. Alloy. compd. Vol. 524, pp. 69.

Macdonald, J.R. (1956). Spin exchange effects in ferromagnetic resonance. Phys. Rev. Vol. 103, pp. 280.

Maciá, F.; Warnicke, P.; Bedau, D.; Im, M.Y.; Fischer, P.; Arena, D.A.; Kent, A.D. (2012). Perpendicular magnetic anisotropy in ultrathin $\mathrm{Co}_{9} \mathrm{Ni}$ multilayer films studied with ferromagnetic resonance and magnetic x-ray microspectroscopy. J. Magn. Magn. Mater. Vol. 324, pp. 3629.

Maklakov, S.S.; Maklakov, S.A.; Ryzhikov, I.A.; Rozanov, K.N.; Osipov, A.V. (2012). Thin Co films with tunable ferromagnetic resonance frequency. J. Magn. Magn. Mater. Vol. 324, pp. 2108.

Maksymowich, L.J.; Sendorek, D. (1983). Surface modes in magnetic thin amorphous films of GdCoMo alloys. J. Magn. Magn. Mater. Vol. 37, pp. 177. 
Maksymowicz, L.J.; Sendorek, D.; Żuberek, R. (1985). Surface anisotropy energy of thin amorphous magnetic films of (Gd1-xCox)1-yMoy alloys; experimental results. J. Magn. Magn. Mater. Vol. 46, pp. 295. Maksymowicz, L.J.; Jankowski, H. (1992). FMR experiment in multilayer structure of FeBSi/Pd. J. Magn. Magn. Mater. Vol. 109, pp. 341.

Marin, C.N. (2006). Thermal and particle size distribution effects on the ferromagnetic resonance in magnetic fluids. J. Magn. Magn. Mater. Vol. 300, pp. 397.

Mastrogiacomo, G.; Fischer H.; Garcia-Rubio, I.; Gehring, A.U. (2010). Ferromagnetic resonance spectroscopic response of magnetite chains in a biological matrix. J. Magn. Magn. Mater. Vol. 322, pp. 661.

Mazur, P.; Mills, D.L. (1982). Inelastic scattering of neutrons by surface spin waves on ferromagnets. Phys. Rev. B. Vol. 26, pp. 5175. McMichael, R.D.; Wigen, P.E. (1990). High power FMR without a degenerate spin wave manifold. Phys. Rev. Lett. Vol. 64, pp. 64.

Meiklejohn, W.H.; Bean, C.P. (1956). New magnetic anisotropy. Phys. Rev. Vol. 102, pp. 1413.

Meiklejohn, W.H.; Bean, C.P. (1957). New magnetic anisotropy. Phys. Rev. Vol. 105, pp. 904.

Mercereau, J.E.; Feynman, R.P. (1956). Physical conditions for ferromagnetic resonance. Phys. Rev. Vol. 104, pp. 63.

Mibu, K.; Nagahama, T.; Ono, T.; Shinjo, T. (1997). Magnetoresistance of quasi-Bloch-wall induced in NiFe/CoSm exchange. J. Magn. Magn. Mater. Vol. 177-181, pp. 1267.

Mibu, K.; Nagahama, T.; Shinjo, T.; Ono, T. (1998). Magnetoresistance of Bloch-wall-type magnetic structures induced in NiFe/CoSm exchange-spring bilayers. Phys. Rev. B. Vol. 58, pp. 6442.

Mills, D.L.; Bland, J.A.C. ( 2006). Nanomagnetism ultrathin films, multilayers and nanostructures. Elsevier B.V. Mills, D.L. (2003). Ferromagnetic resonance relaxation in ultrathin metal films: The role of the conduction electrons. Phys. Rev. B. Vol. 68, pp. 014419.

Min, J.H.; Cho, J.U.; Kim, Y.K.; Wu, J.H.; Ko, Y.D.; Chung, J.S. (2006). Substrate effects on microstructure and magnetic properties of electrodeposited Co nanowire arrays. J. Appl. Phys. Vol. 99, pp. 08Q510.

Miyake, K.; Noh, S.M.; Kaneko, T.; Imamura, H.; Sahashi, M. (2012). Study on highfrequency $3-\mathrm{D}$ magnetization precession modes of circular magnetic nano-dots using coplanar wave guide vector network analyzer ferromagnetic resonance. IEEE T. Magn. Vol. 48, pp. 1782.

Mizukami, S.; Nagashima, S.; Yakata, S.; Ando, Y.; Miyazaki, T. (2007). Enhancement of DC voltage generated in ferromagnetic resonance for magnetic thin film. J. Magn. Magn. Mater. Vol. 310, pp. 2248.

Morrish, A. H. (1965).The physical principles of magnetism Wiley, New York.

Nagamine, L.C.C.M.; Geshev, J.; Menegotto, T.; Fernandes, A.A.R.; Biondo, A.; Saitovitch, E.B. (2005). Ferromagnetic resonance and magnetization studies in exchange-coupled NiFe/Cu/NiFe structures. J. Magn. Magn. Mater. Vol. 288, pp. 205.

Nakai, T.; Yamaguchi, M.; Kikuchi, H.; Iizuka, H.; Arai, K.I. (2002). Remarkable improvement of sensitivity for high-frequency carrier-type magnetic field sensor with ferromagnetic resonance. J. Magn. Magn. Mater. Vol. 242-245, pp. 1142. 
Neudecker, I.; Woltersdorf, G.; Heinrich, B.; Okuno, T.; Gubbiotti, G.; Back, C.H. (2006). Comparison of frequency, field, and time domain ferromagnetic resonance methods. J. Magn. Magn. Mater. Vol. 307, pp. 148.

Olive, E.; Lansac, Y.; Wegrowe, J.E. (2012). Beyond ferromagnetic resonance: The inertial regime of the magnetization. Appl. Phys. Lett. Vol. 100, pp. 192407.

Osthöver, C.; Grünberg, P.; Arons, R.R. (1998). Magnetic properties of doped La0.67Ba0.33Mn1$\mathrm{yA}_{y} \mathrm{O}_{3}, \mathrm{~A}-\mathrm{Fe}, \mathrm{Cr}$. J. Magn. Magn. Mater. Vol. 177-181, pp. 854.

Owens, F.J. (2009). Ferromagnetic resonance observation of a phase transition in magnetic field-aligned $\mathrm{Fe}_{2} \mathrm{O}_{3}$ nanoparticles. J. Magn. Magn. Mater. Vol. 321, pp. 2386.

Öner, Y.; Özdemir, M.; Aktaş, B.; Topacli, C.; Haris, E.A.; Senoussi, S. (1997). The role of Pt impurities on both bulk and surface anisotropies in amorphous NiMn films. J. Magn. Magn. Mater. Vol. 170, pp. 129.

Özdemir, M.; Aktaş, B.; Öner, Y.; Sato, T.; Ando, T. (1996). A spin- wave resonance study on reentrant Mn77Mn23 thin films. J. Magn. Magn. Mater. Vol. 164, pp. 53.

Özdemir, M.; Aktaş, B.; Öner, Y.; Sato, T.; Ando, T. (1997). Anomalous anisotropy of reentrant Niz7Mn23 film. J. Phys.: Condens. Matter. Vol. 9, pp. 6433.

Özdemir, M.; Öner, Y.; Aktaş, B. (1998). Evidence of superparamagnetic behaviour in an amorphous Ni62 Mn38 film by ESR measurements. Phys. B. Vol. 252, pp. 138.

Patel, R.; Owens, F.J. (2012). Ferromagnetic resonance and magnetic force microscopy evidence for above room temperature ferromagnetism in Mn doped Si made by a solid state sintering process. Solid State Commun. Vol. 152, pp. 603.

Patton, C.E.; Hurben, M.J. (1995). Theory of magnetostatic waves for in-plane magnetized isotropic films. J. Magn. Magn. Mater. Vol. 139, pp. 263. Patton, C.E.; Hurben, M.J. (1996). Theory of magnetostatic waves for in-plane magnetized anisotropic films. J. Magn. Magn. Mater. Vol. 163, pp. 39.

Park, D.G.; Kim, C.G.; Kim, W.W.; Hong, J.H. (2007). Study of GMI-valve characteristics in the Co-based amorphous ribbon by ferromagnetic resonance. J. Magn. Magn. Mater. Vol. 310, pp. 2295.

Parkin, S.S.P.; More, N.; Roche, K.P. (1990). Oscillations in exchange coupling and magneto resistance in metallic superlattice structures: $\mathrm{Co} / \mathrm{Ru}, \mathrm{Co} / \mathrm{Cr}$, and $\mathrm{Fe} / \mathrm{Cr}$. Phys. Rev. Lett. Vol. 64, pp. 2304.

Parkin, S.S.P.; Bhadra, R.; Roche, K.P. (1991(a)). Oscillatory magnetic exchange coupling through thin copper layers. Phys. Rev. Lett. Vol. 66, pp. 2152.

Parkin, S.S.P. (1991(b)). Systematic variation of the strength and oscillation period of indirect magnetic exchange coupling through the $3 d, 4 d$, and $5 d$ transition metals. Phys. Rev. Lett. Vol. 67, pp. 3598.

Parkin, S.S.P.; Farrow, R.F.C.; Marks, R.F.; Cebollada, A.; Harp, G.R.; Savoy, R.J. (1994). Oscillations of interlayer exchange coupling and giant magnetoresistance in (111) oriented permalloy/Au multilayers. Phys. Rev. Lett. Vol. 72, pp. 3718.

Parvatheeswara, R.B.; Caltun, O.; Dumitru, I.; Spinu, L. (2006). Ferromagnetic resonance parameters of ball-milled Ni-Zn ferrite nanoparticles. J. Magn. Magn. Mater. Vol. 304, pp. 752. 
Paul, A.; Bürgler, D.E.; Grünberg, P. (2005). Enhanced exchange bias in ferromagnet/ antiferromagnet multilayers. J. Magn. Magn. Mater. Vol. 286, pp. 216.

Paz, E.; Cebollada, F.; Palomares, F.J.; González, J.M.; Martins, J.S.; Santos, N.M., Sobolev, N.A. (2012). Ferromagnetic resonance and magnetooptic study of submicron epitaxial Fe(001) stripes. J. Appl. Phys. Vol. 111, pp. 123917.

Pires, M.J.M.; Mansanares, A.M., da Silva, E.C.; Schmidt, J.E.; Meckenstock, R.; Pelzl, J. (2006). Ferromagnetic resonance studies in granular CoCu codeposited films. J. Magn. Magn. Mater. Vol. 300, pp. 382.

Platow, W.; Anisimov, A.N.; Dunifer, G.L.; Farle, M.; Baberschke, K. (1998). Correlations between ferromagnetic-resonance linewidths and sample quality in the study of metallic ultrathin films. Phys. Rev. B. Vol. 58, pp. 5611.

Pollmann, J.; Srajer, G.; Hakel, D.; Lang, J.C.; Maser, J.; Jiang, J.S.; Bader, S.D. (2001). Magnetic imaging of a buried SmCo layer in a spring magnet. J. Appl. Phys. Vol. 89, pp. 7165 .

Poole, C.P.; Owens J.F. (2003). Introduction to nanotechnology. John Wiley \& Sons, Inc. ISBN: 0-471-07935-9.

Prieto, A.G.; Fdez-Gubieda, M.L.; Lezama, L.; Orue, I. (2012). Study of surface effects on $\mathrm{CoCu}$ nanogranular alloys by ferromagnetic resonance. J. Appl. Phys. Vol. 111, pp. $07 \mathrm{C} 105$.

Purcell, E.M.; Torrey, H.C.; Pound, R.V. (1946). Resonance absorption by nuclear magnetic moments in a solid. Phys. Rev. Vol. 69, pp. 37.

Puszkarski, H. (1992). Spectrum of interface coupling-affected spin-wave modes in ferromagnetic bilayer films. Phys. stat. sol. Vol. 171, pp. 205.

Rabi, I.I.; Zacharias, J.R.; Millman, S.; Kusch, P. (1938). A new method of measuring nuclear magnetic moment, Phys. Rev. Vol. 53, pp. 318.

Rachford, F.J.; Vittoria, C. (1981). Ferromagnetic anti-resonance in non-saturated magnetic metals. J. Appl. Phys. Vol. 52, pp. 2253.

Rado, G.T. (1958). Effect of electronic mean free path on spin-wave resonance in ferromagnetic metals. J. Appl. Phys. Vol. 29, pp. 330. Raikher, Yu.L.; Stepanov, V.I. (2003). Nonlinear dynamic response of superparamagnetic nanoparticles. Microelectron. Eng. Vol. 69, pp. 317.

Raita, O.; Popa, A.; Stan, M.; Suciu, R.C.; Biris, A.; Giurgiu, L.M. (2012). Effect of Fe concentration in $\mathrm{ZnO}$ powders on ferromagnetic resonance spectra. Appl. Magn. Reson. Vol. 42, pp. 499.

Rahman, F. (2008). Nanostructures in electronics and photonics. Pan Stanford Publishing Pte. Ltd. Singapore, 596224.

Rameev, B.Z.; Aktaş, B.; Khaibullin, R.I.; Zhikharev, V.A.; Osin, Yu.N.; Khaibullin, I.B. (2000). Magnetic properties of iron-and cobalt-implanted silicone polymers. Vacuum. Vol. 58, pp. 551.

Rameev, B.Z.; Yıldız, F.; Aktaş, B.; Okay, C.; Khaibullin, R.I.; Zheglov, E.P.; Pivin, J.C.; Tagirov, L.R. (2003(a)). I on synthesis and FMR studies of iron and cobalt nanoparticles in polyimides. Microelectron. Eng. Vol. 69, pp. 330. 
Rameev, B.Z.; Yilgin, R.; Aktaş, B.; Gupta, A.; Tagirov, L.R. (2003(b)). FMR studies of CrO epitaxial thin films. Microelectron. Eng. Vol. 69, pp. 336.

Rameev, B.Z.; Gupta, A.; Anguelouch, A.; Xiao, G.; Yıldız, F.; Tagirov, L.R.; Aktaş, B. (2004 (b)). Probing magnetic anisotropies in half-metallic $\mathrm{CrO}_{2}$ epitaxial films by FMR. $J$. Magn. Magn. Mater. Vol. 272-276, pp. 1167.

Rameev, B.; Okay, C.; Yıldız, F.; Khaibullin, R.I.; Popok, V.N.; Aktas, B. (2004 (a)). Ferromagnetic resonance investigations of cobalt-implanted polyimides. J. Magn. Magn. Mater. Vol. 278, pp. 164.

Ramesh, M.; Wigen, P.E. (1988 (a)). Ferromagnetic resonance of parallel stripe domainsdomain wall system. J. Magn. Magn. Mater. Vol. 74, pp. 123. Ramesh, M.; Ren, E.W.; Artman, J.O.; Kryder, M.H. (1988 (b)). Domain mode ferromagnetic resonance studies in bismuth-substituted magnetic garnet films. J. Appl. Phys. Vol. 64, pp. 5483.

Ramprasad, R.; Zurcher P.; Petras, M.; Miller, M.; Renaud, P. (2004). Magnetic properties of metallic ferromagnetic nanoparticle composites. J. Appl. Phys. Vol. 96, pp. 519.

Ramsey, N.F. (1985). Molecular beams. Oxford University Press. New York.

Raposo, V.; Zazo, M.; Iñiguez, J. (2011). Comparison of ferromagnetic resonance between amorphous wires and microwires. J. Magn. Magn. Mater. Vol. 323, pp. 1170.

Reich, K.H. (1955). Ferromagnetic resonance absorption in a nickel single crystal at low temperature. Phys. Rev. Vol. 101, pp. 1647.

Rezende, S.M.; Moura, J.A.S.; de Aguiar, F.M. (1993). Ferromagnetic resonance in Ag coupled Ni films. J. Appl. Phys. Vol. 73, pp. 6341.

Riedling, S.; Knorr, N.; Mathieu, C.; Jorzick, J.; Demokritov, S.O.; Hillebrands, B.; Schreiber, R.; Grünberg, P. (1999). Magnetic ordering and anisotropies of atomically layered Fe/Au(001) multilayers. J. Magn. Magn. Mater. Vol.198-199, pp. 348.

Richard, C.; Houzet, M.; Meyer, J.S. (2012). Andreev current induced by ferromagnetic resonance. Appl. Phys. Lett. Vol. 109, pp. 057002.

Rivkin, K.; Xu, W., De Long, L.E.; Metlushko, V.V.; Ilic, B.; Ketterson, J.B. (2007). Analysis of ferromagnetic resonance response of square arrays of permalloy nanodots. J. Magn. Magn. Mater. Vol. 309, pp. 317.

Rodbell, D.S. (1964). Ferromagnetic resonance absorption linewidth of nickel metal. Evidence for Landau- Lifshitz damping. Phys. Rev. Lett. Vol. 13, pp. 471.

Rook, K.; Artman, J.O. (1991). Spin wave resonance in FeAlN films. IEEE Trans. Magn. Vol. 27, pp. 5450. Roy, W.V.; Boeck, J.D.; Borghs, G. (1992). Optimization of the magnetic field of perpendicular ferromagnetic thin films for device applications. Appl. Phys. Lett. Vol. 61, pp. 3056.

Rousseau, O.; Viret, M. (2012). Interaction between ferromagnetic resonance and spin currents in nanostructures. Phys. Rev. B. Vol. 85, pp. 144413.

Römer, F.M.; Möller, M.; Wagner, K.; Gathmann, L.; Narkowicz, R.; Zähres, H.; Salles, B.R.; Torelli, P.; Meckenstock, R.; Lindner, J.; Farle, M. (2012). In situ multifrequency ferromagnetic resonance and x-ray magnetic circular dichroism investigations on Fe/GaAs(110): Enhanced g-factor. J. Appl. Phys. Vol. 100, pp. 092402. 
Ruderman, M.A.; Kittel, C. (1954). Indirect exchange coupling of nuclear magnetic moments by conduction electrons. Phys. Rev. Vol. 96, pp. 99. Rusek, P. (2004). Spin dynamics of ferromagnetic spin glass. J. Magn. Magn. Mater. Vol. 272-276, pp. 1332.

Sarmiento, G.; Fdez-Gubieda, M.L.; Siruguri, V.; Lezama, L.; Orue, I. (2007). Ferromagnetic resonance study of $\mathrm{Fe}_{50} \mathrm{Ag} 50$ granular film. J. Magn. Magn. Mater. Vol. 316, pp. 59.

Sasaki, M.; Jönsson, P.E.; Takayama, H.; Mamiya, H. (2005). Aging and memory effects in superparamagnets and superspin glasses. Rev. B. Vol. 71, pp. 104405.

Schäfer, S.; Pachauri, N.; Mewes, C.K.A.; Mewes, T.; Kaiser, C.; Leng, Q.; Pakala, M. (2012). Frequency-selective control of ferromagnetic resonance linewidth in magnetic multilayers. J. Appl. Phys. Vol. 100, pp. 032402.

Schmool, D.S.; Barandiarán, J.M. (1998). Ferromagnetic resonance and spin wave resonance in multiphase materials: theoretical considerations. J. Phys.: Condens. Matter. Vol. 10, pp. 10679.

Schmool, D.S.; Schmalzl, M. (2007). Ferromagnetic resonance in magnetic nanoparticle assemblies. J. Non-Cryst. Solids. Vol. 353, pp. 738.

Scholz, W.; Suess, D.; Schrefl, T.; Fidler, J. (2000). Micromagnetic simulation of structureproperty relations in hard and soft magnets. Comp. Mater. Sci. Vol. 18, pp. 1.

Schreiber, F.; Frait, Z. (1996). Spinwave resonance in high conductivity films: The Fe-Co alloy system. Phys. Rev. B. Vol. 54, pp. 6473. Schrefl, T.; Kronmüller, H.; Fidler, J. (1993(a)). Exchange hardening in nano-structured permanent magnets. J. Magn. Magn. Mater. Vol. 127, pp. 273.

Schrefl, T.; Schmidts, H.F.; Fidler, J.; Kronmüller, H. (1993(b)). The role of exchange and dipolar coupling at grain boundaries in hard magnetic materials. J. Magn. Magn. Mater. Vol. 124, pp. 251. Schrefl, T.; Fidler, J. (1998). Modelling of exchange-spring permanent magnets. J. Magn. Magn. Mater. Vol. 177-181, pp. 970.

Schrefl, T.; Forster, H.; Fidler, J.; Dittrich, R.; Suess, D.; Scholz, W. (2002). Magnetic hardening of exchange spring multilayers. Proof XVII Rare Earth Magnets Workshop, University of Delaware, ed: Hadjipanayis, G.; Bonder M.J. pp. 1006.

Schultz, S.; Gullikson, E.M. (1983). Measurement of static magnetization using electron spin resonance. Rev. Sci. Insrum. Vol. 54, pp. 1383.

Seemann, K.; Leiste, H.; Klever, C. (2009). On the relation between the effective ferromagnetic resonance linewidth $\Delta \mathrm{f}_{\text {eff }}$ and damping parameter $\alpha_{\text {eff }}$ in ferromagnetic Fe-Co-Hf-N nanocomposite films. J. Magn. Magn. Mater. Vol. 321, pp. 3149.

Seemann, K.; Leiste, H.; Klever, Ch. (2010). Determination of intrinsic FMR line broadening in ferromagnetic $\left(\mathrm{Fe}_{44} \mathrm{C}_{056}\right)_{77} \mathrm{Hf}_{12} \mathrm{~N}_{11}$ nanocomposite films. J. Magn. Magn. Mater. Vol. 322, pp. 2979.

Seib, J.; Steiauf, D.; Fähnle, M. (2009).Linewidth of ferromagnetic resonance for systems with anisotropic damping. Phys. Rev. B. Vol. 79, pp. 092418.

Sellmyer, D.; Skomski, R. (2006). Advanced magnetic nanostructures. Springer Science+Business Media, Inc.

Sihues, M.D.; Durante-Rincón, C.A.; Fermin, J.R. (2007). A ferromagnetic resonance study of NiFe alloy thin films. J. Magn. Magn. Mater. Vol. 316, pp. 462. 
Singh, A.; Chowdhury, P.; Padma, N.; Aswal, D.K.; Kadam, R.M.; Babu, Y.; Kumar, M.L.J.; Viswanadham, C.S.; Goswami, G.L.; Gupta, S.K.; Yakhmi, J.V. (2006). Magnetotransport and ferromagnetic resonance studies of polycrystalline $\mathrm{La}_{0.6} \mathrm{~Pb}_{0.4 \mathrm{NO}} \mathrm{NO}_{3}$ thin films. Solid State Commun. Vol. 137, pp. 456.

Shames, A.I.; Rozenberg, E.; Sominski, E.; Gedanken, A. (2012). Nanometer size effects on magnetic order in La1-x CaxMnO3 ( $\mathrm{x}=50.5$ and 0.6$)$ manganites, probed by ferromagnetic resonance. J. Appl. Phys. Vol. 111, pp. 07D701.

Sklyuyev, A.; Ciureanu, M.; Akyel, C.; Ciureanu, P.; Yelon, A. (2009). Microwave studies of magnetic anisotropy of Co nanowire arrays. J. App. Phys. Vol. 105, pp. 023914.

Skomski, R.; Coey, J.M.D. (1993). Giant energy product in nanostructured two-phase magnets. Phys. Rev. B. Vol. 48, pp. 15812.

Skomski, R. (2008) .Simple models of magnetism. Oxford University Press. ISBN 978-0-19857075-2.

Slichter, C.P. (1963). Principle of Magnetic Resonance. Harper \& Row, New York. Smit, J.; Beljers, H. G. (1955). Ferromagnetic resonance absorption in $\mathrm{BaFe}_{2} \mathrm{O}_{19}$, a high anisotropy crystal. Philips Res. Rep. Vol. 10, pp. 113.

Spaldin, N.A. (2010). Magnetic materials: Fundamentals and applications. Cambridge University Press. ISBN 139780521886697.

Sparks, M. (1964). Ferromagnetic Relaxation Theory. McGraw-Hill, New York.

Sparks, M. (1969). Theory of surface spin pinning in ferromagnetic resonance. Phys. Rev. Lett. Vol. 22, pp. 1111.

Sparks, M. (1970(a)). Ferromagnetic resonance in thin films. III. Theory of mode Intensities. Phys. Rev. B. Vol. 1, pp. 3869.

Sparks, M. (1970(b)). Ferromagnetic resonance in thin films I and II. Phys. Rev. B. Vol. 1, pp. 3831. Sparks, M. (1970(c)). Ferromagnetic resonance in thin films. I. Theory of normalmode frequencies. Phys. Rev. B. Vol. 1, pp. 3831.

Sparks, M. (1970(d)). Ferromagnetic resonance in thin films. I. Theory of normal-mode intensities. Phys. Rev. B. Vol. 1, pp. 3869.

Speriosu, V.S.; Parkin, S.S.P. (1987). Standing spin waves in FeMn/NiFe/FeMn exchange bias structures. IEEE Trans. magn. Vol. 23, pp. 2999.

Spinu, L.; Dumitru, I.; Stancu, A.; Cimpoesu, D. (2006). Transverse susceptibility as the lowfrequency limit of ferromagnetic resonance. J. Magn. Magn. Mater. Vol. 296, pp. 1.

Spoddig, D.; Meckenstock, R.; Bucher, J.P.; Pelzl, J. (2005). Studies of ferromagnetic resonance line width during electrochemical deposition of Co films on $\mathrm{Au}\left(\begin{array}{lll}1 & 1 & 1\end{array}\right)$. J. Magn. Magn. Mater. Vol. 286, pp. 286.

Song, Y.Y.; Kalarickal, S.; Patton, C.E. (2003). Optimized pulsed laser deposited barium ferrite thin films with narrow ferromagnetic resonance linewidths. J. Appl. Phys. Vol. 94, pp. 5103.

Song, H.; Mulley, S.; Coussens, N.; Dhagat, P.; Jander, A.; Yokochi, A. (2012). Effect of packing fraction on ferromagnetic resonance in $\mathrm{NiFe}_{2} \mathrm{O}_{4}$ nanocomposites. J. Appl. Phys. Vol. 111, pp. 07E348.

Stashkevich, A.A.; Roussigné, Y.; Djemia, P.; Chérif, S.M.; Evans, P.R.; Murphy, A.P.; Hendren, W.R.; Atkinson, R.; Pollard, R.J.; Zayats, A.V.; Chaboussant, G.; Ott, F. (2009). 
Spin-wave modes in Ni nanorod arrays studied by Brillouin light scattering. Phys. Rev. B. Vol. 80, pp. 144406.

Stoner, E.C.; Wohlfarth, E. P. (1948). A mechanism of magnetic hysteresis in heterogeneous alloys. Phil. Trans. R. Soc. A. Vol. 240, pp. 599.

Sun, Y.; Duan, L.; Guo, Z.; DuanMu, Y.; Ma, M.; Xu, L.; Zhang, Y.; Gu, N. (2005). An improved way to prepare superparamagnetic magnetite-silica core-shell nanoparticles for possible biological application. J. Magn. Magn. Mater. Vol. 285, pp. 65.

Sun, Y.; Song, Y.Y.; Chang, H.; Kabatek, M.; Jantz, M.; Schneider, W.; Wu, M.; Schultheiss, H.; Hoffmann, A. (2012(a)). Growth and ferromagnetic resonance properties of nanometer-thick yttrium iron garnet films. Appl. Phys. Lett. Vol. 101, pp. 152405.

Sun, Y.; Song Y.Y.; Wu, M. (2012(b)). Growth and ferromagnetic resonance of yttrium iron garnet thin films on metals. Appl. Phys. Lett. Vol. 101, pp. 082405.

Sun, L.; Wang, Y.; Yang, M.; Huang, Z.; Zhai, Y.; Xu, Y.; Du, J.; Zhai, H. (2012(c)). Ferromagnetic resonance studies of $\mathrm{Fe}$ thin films with dilute heavy rare-earth impurities. J. Appl. Phys. Vol. 111, pp. 07A328.

Suzuki, Y.; Katayama, T.; Takanashi, K.; Schreiber, R.; Gtinberg, P.; Tanaka, K. (1997) . The magneto-optical effect of $\mathrm{Cr}(001)$ wedged ultrathin films grown on $\mathrm{Fe}(001)$. J. Magn. Magn. Mater. Vol. 165, pp. 134.

Szlaferek, A. (2004). Model exchange-spring nanocomposite. Status Solidi B, Vol. 241, pp. 1312.

Tannenwald, P.E.; Seawey, M.H. (1957). Ferromagnetic resonance in thin films of permalloy. Phys. Rev. Vol. 105, pp. 377.

Tartaj, P.; González-Carreño, T.; Bomati-Miguel, O.; Serna, C. J. (2004). Magnetic behavior of superparamagnetic Fe nanocrystals confined inside submicron-sized spherical silica particles. Phys. Rev. B. Vol. 69, pp. 094401.

Teale, R.W.; Pelegrini, F. (1986). Magnetic surface anisotropy and ferromagnetic resonance in the single crystal GdAl. J. Phys. F:Met. Phys. Vol. 16, pp. 621.

Terry, E.M. (1917). The magnetic properties of iron, nickel and cobalt above the curie point, and Keeson's quantum theory of magnetism. Phys. Rev. Vol. 9, pp. 394.

Topkaya, R.; Erkovan, M.; Öztürk, A.; Öztürk, O.; Aktaş, B.; Özdemir, M. (2010). Ferromagnetic resonance studies of exchange coupled ultrathin $\mathrm{Py} / \mathrm{Cr} / \mathrm{Py}$ trilayers. $J$. Appl. Phys. Vol. 108, pp. 023910.

Tsai, C.C.; Choi, J.; Cho, S.; Lee, S.J.; Sarma, B.K.; Thompson, C.; Chernyashevskyy, O.; Nevirkovets, I.; Metlushko, V.; Rivkin, K.; Ketterson, J.B. (2009). Vortex phase boundaries from ferromagnetic resonance measurements in a patterned disc array. Phys. Rev. B. Vol. 80, pp. 014423.

Uhlenbeck, G.E.; Goudsmit,S. (1925). Ersetzung der hypothese vom unmechanischen zwang durch eine forderung bezüglich des inneren verhaltens jedes einzelnen elektrons. Die Naturwissenschaften. Vol. 13, Issue (47), pp. 953.

Valenzuela, R.; Zamorano, R.; Alvarez, G.; Gutiérrez, M.P.; Montiel, H. (2007). Magnetoimpedance, ferromagnetic resonance, and low field microwave absorption in amorphous ferromagnets. J. Non-Cryst. Solids. Vol. 353, pp. 768. 
Valenzuela, R.; Herbst, F.; Ammar, S. (2012). Ferromagnetic resonance in Ni-Zn ferrite nanoparticles in different aggregation states. J. Magn. Magn. Mater. Vol. 324, pp. 3398.

Van Vleck, J.H. (1950). Concerning the theory of ferromagnetic resonance absorption. Phys. Rev. Vol. 78, pp. 266.

Vargas, J.M.; Zysler, R.D.; Butera, A. (2007). Order-disorder transformation in FePt nanoparticles studied by ferromagnetic resonance. Appl. Surf. Sci. Vol. 254, pp. 274.

Vilasi, D. (2001). Hamiltonian dynamics. World Scientific. ISBN 981-02-3308-6.

Vittoria, C. (1993). Microwave Properties in Magnetic Films. World Scientific, Singapore. pp. 87.

Vlasko-Vlasov, K.; Welp, U.; Jiang, J.S.; Miller, D.J.; Crabtree, G.W.; Bader, S.D. (2001). Field induced biquadratic exchange in hard/soft ferromagnetic bilayers. Phys. Rev. Lett. Vol. 86, pp. 4386.

Voges, F.; de Gronckel, H.; Osthöver, C.; Schreiber, R.; Grünberg, P. (1998). Spin valves with $\mathrm{CoO}$ as an exchange bias layer. J. Magn. Magn. Mater. Vol. 190, pp. 183.

Vounyuk, B.P.; Guslienko, K.Y.; Kozlov, V.I.; Lesnik, N.A.; Mitsek, A.I. (1991). Effect on the interaction of layers on a ferromagnetic resonance in two layer feromagnetic films. Sov. Phys. Solid State. Vol. 33, pp. 250.

Walker, L.R. (1957). Magnetostatic modes in ferromagnetic resonance. Phys. Rev. Vol. 105, pp. 390.

Wallis, T.M.; Moreland, J.; Riddle, B.; Kabos, P. (2005). Microwave power imaging with ferromagnetic calorimeter probes on bimaterial cantilevers. J. Magn. Magn. Mater. Vol. 286, pp. 320.

Wang, X.; Deng, L.J.; Xie, J.L.; Li, D. (2011). Observations of ferromagnetic resonance modes on FeCo-based nanocrystalline alloys. J. Magn. Magn. Mater. Vol. 323, pp. 635.

Weil, J.A.; Bolton, J.R.; Wertz, J.E. (1994). Electron Paramagnetic Resonance: elementary Theory and Practical Application. John Wiley-Sons. New York. Weil, J.A.; Bolton, J.R. (2007). Electron paramagnetic resonance. John Wiley E Sons, Inc. Hoboken, New Jersey. ISBN 978-0471-75496-1.

Weiss, M.T.; Anderson, P.W. (1955). Ferromagnetic resonance in ferroxdure. Phys. Rev. Vol. 98, pp. 925.

Wegrowe, J.E.; Kelly, D.; Franck, A.; Gilbert, S.E.; Ansermet, J.Ph. (1999). Magnetoresistance of ferromagnetic nanowires. Phys. Rev. Lett. Vol. 82, pp. 3681.

Wegrowe, J.E.; Comment, A.; Jaccard, Y.; Ansermet, J.Ph. (2000). Spin-dependent scattering of a domain wall of controlled size. Phys. Rev. B. Vol. 61, pp. 12216.

White, R.L.; Solt, I.H. (1956). Multiple ferromagnetic resonance in ferrite spheres. Phys. Rev. Vol. 104, pp. 56.

Wiekhorst, F.; Shevchenko, E.; Weller, H.; Kötzler, J. (2003). Anisotropic superparamagnetism of mono dispersive cobalt-platinum nanocrystals. Phys. Rev. B. Vol. 67, pp. 224416.

Wigen P.E.; Zhang, Z. (1992). Ferromagnetic resonance in coupled magnetic multilayer systems. Braz. J. Phys. Vol. 22, pp. 267. Wigen, P.E.; Kooi, C.F.; Shanaberger, M.R.; Rosing, T.R. (1962). Dynamic pinning in thin-film spin-wave resonance. Phys. Rev. Lett. 
Vol. 9, pp. 206. Wigen, P.E. (1984). Microwave properties of magnetic garnet thin films. Thin Solid Films. Vol. 114, pp. 135.

Wigen, P.E.; Zhang, Z.; Zhou, L.; Ye, M.; Cowen, J.A. (1993). The dispersion relation in antiparallel coupled ferromagnetic films. J. Appl. Phys. Vol. 73, pp. 6338.

Wigen, P.E. (1998). Routes to chaos in ferromagnetic resonance and the return trip: Controlling and synchronizing Chaos, in Bar'yakhtar, V.G.; Wigen, P.E.; Lesnik, N.A. (Eds.). Frontiers in magnetism of reduced dimension systems Nato ASI series (Kluwer, Dordrecht) pp. 29. Wojtowicz, T. (2005). Ferromagnetic resonance study of the free-hole contribution to magnetization and magnetic anisotropy in modulation-doped Ga1×MnxAs/Ga1-yAlyAs: Be. Phys. Rev. B. Vol. 71, pp. 035307.

Wolfram, T.; De Wames, R.E. (1971). Magneto-exchange branches and spin-wave resonance in conducting and insulating films: Perpendicular resonance. Phys. Rev. B. Vol. 4, pp. 3125 .

Woltersdorf, G.; Heinrich, B.; Woltersdorf, J.; Scholz, R. (2004). Spin dynamics in ultrathin film structures with a network of misfit dislocations. Journal of Applied Physics. Vol. 95, pp. 7007-7009.

Woods, S.I.; Kirtley, J.R.; Sun, S.; Koch, R.H. (2001). Direct investigation of superparamagnetism in Co nanoparticle films. Phys. Rev. Lett. Vol. 87, pp. 137205.

Wüchner, S.; Toussaint, J.C.; Voiron, J. (1997). Magnetic properties of exchange-coupled trilayers of amorphous rare-earth-cobalt alloys. J. Phys. Rev. B. Vol. 55, pp. 11576.

Xu, Y.; Zhang, D.; Zhai, Y.; Chen, J.; Long, J.G.; Sang, H.; You, B.; Du, J.; Hu, A.; Lu, M.; Zhai, H.R. (2004). FMR study on magnetic thin and ultrathin Ni-Fe films. Phys. Stat. Sol. (c). Vol. 12, pp. 3698.

Yalçın, O.; Yıldız, F.; Özdemir, M.; Aktaş, B.; Köseoğlu, Y.; Bal, M.; Touminen, M.T. (2004(a)). Ferromagnetic resonance studies of Co nanowire arrays. J. Magn. Magn. Mater. Vol. 272-276, pp. 1684.

Yalçın, O.; Yıldız, F.; Özdemir, M.; Rameev, B.; Bal, M.; Tuominen, M.T. (2004(b)). FMR Studies of Co Nanowire Arrays, Nanostructures Magnetic Materials and Their Applications. Kluwer Academic Publisher. Nato Science Series. Mathematics, Physics and Chemistry. Vol. 143, pp. 347.

Yalçın, O. (2004(c)). PhD Thesis, investigation of phase transition in inorganic spin-Peierls $\mathrm{CuGeO}_{3}$ systems by ESR techgnique. Gebze institute of technology, 2004 Gebze, Kocaeli, Turkey.

Yalçın, O.; Aktaş, B. (2003). The Effects of $\mathrm{Zn}^{2+}$ _doping on Spin-Peierls transition in $\mathrm{CuGeO}_{3}$ J. Magn. Magn. Mater. Vol. 258-259, pp. 137.

Yalçın, O.; Yıldız, F.; Aktaş, B. (2007(a)). Spin-flop and spin-Peierls transition in doped $\mathrm{CuGeO}_{3}$ Spectrochim. Acta Part A. Vol. 66, pp. 307.

Yalçın, O. (2007(b)). Comparison effects of different doping on spin-Peierls transition in $\mathrm{CuGeO}_{3}$ Spectrochim. Acta Part A. Vol. 68, pp. 1320.

Yalçın, O.; Kazan, S.; Şahingöz, R.; Yildiz, F.; Yerli, Y.; Aktaş, B. (2008(a)). Thickness dependence of magnetic properties of Co90Fe10 nanoscale thin films. J. Nanosci. Nanotech. Vol. 8, pp. 841 . 
Yalçın, O.; Erdem, R.; Övünç, S. (2008(b)). Spin-1 model of noninteracting nanoparticles. Acta Phys. Pol. A. Vol. 114, pp. 835. Yalçın, O.; Erdem,R.; Demir, Z. (2012). Magnetic properties and size effects of spin-1/2 and spin- 1 models of core-surface nanoparticles in different type lattices, smart nanoparticles technology, Abbass Hashim (Ed.), ISBN: 978953-51-0500-8, InTech, DOI: 10.5772/34706. Available from:

http://www.intechopen.com/books/smart-nanoparticles-technology/magneticproperties-and-size-effects-of-spin-1-2-and-spin-1-models-of-core-shell-nanoparticlesin-di.

Yeh, Y.C.; Jin, J.D.; Li, C.M.; Lue, J.T. (2009). The electric and magnetic properties of Co and Fe films percept from the coexistence of ferromagnetic and microstrip resonance or a $\mathrm{T}$ type microstrip. Measurement. Vol. 42, pp. 290.

Yıldız, F.; Yalçın, O.; Özdemir, M.; Aktaş, B.; Köseoğlu, Y.; Jiang, J.S. (2004(a)). Magnetic properties of SmCo/Fe exchange spring magnets. J. Magn. Magn. Mater. Vol. 272-276, pp. 1941.

Yıldız, F.; Yalçın,O.; Aktaş, B.; Özdemir, M.; Jiang, J.S. (2004(b)). Ferromagnetic resonance studies on Sm-Co/Fe thin films. MSMW'04 Symposium Proceedings. Kharkov, Ukraine.

Yıldız, F.; Kazan, S.; Aktas, B.; Tarapov, S.; Samofalov, V.; Ravlik, A. (2004(c)). Magnetic anisotropy studies on $\mathrm{FeNiCo} / \mathrm{Ta} / \mathrm{FeNiCo}$ three layers film by layer sensitive ferromagnetic resonance technique. Phys. stat. sol.(c). Vol. 12, pp. 3694.

Yosida, K. (1957). Magnetic properties of Cu-Mn alloys. Phys. Rev. Vol. 106, pp. 893. Yoshikiyo, M.; Namai, A.; Nakajima, M.; Suemoto, T.; Ohkoshi, S. (2012). Anomalous behavior of high-frequency zero-field ferromagnetic resonance in aluminumsubstituted $\varepsilon$-Fe2O3. J. Appl. Phys. Vol. 111, pp. 07A726.

Young, J.A.; Uehling, E.A. (1953). The tensor formulation of ferromagnetic resonance. Phys. Rev. Vol. 93, pp. 544.

Yu, J.T.; Turk, R.A.; Wigen, P.E. (1975). Exchange dominated surface spinwaves in yttriumiron-garnet films. Phys. Rev. B. Vol. 11, pp. 420.

Zakeri, K.; Kebe, T.; Lindner, J.; Farle, M. (2006). Magnetic anisotropy of Fe/GaAs(001) ultrathin films investigated by in situ ferromagnetic resonance. J. Magn. Magn. Mater. Vol. 299, pp. L1.

Zakeri, Kh.; Lindner, J.; Barsukov, I.; Meckenstock, R.; Farle, M.; von Hörsten, U.; Wende, H.; Keune, W. (2007). Spin dynamics in ferromagnets: Gilbert damping and twomagnon scattering. Phys. Rev. B. Vol. 76, pp. 104416.

Zavoisky, E. (1945). Spin-magnetic resonance in paramagnetics. J. Phys. USSR. Vol. 9, pp. 211.

Zianni, X.; Trohidou, K.N. (1998). Monte carlo simulations the coercive behaviour of oxide coated ferromagnetic particles. J. Phys.: Condens. Matter. Vol. 10, pp. 7475.

Zhai, Y.; Shi, L.; Zhang, W.; Xu, Y.X.; Lu, M.; Zhai, H.R.; Tang, W.X.; Jin, X.F.; Xu, Y.B.; Bland, J.A.C. (2003). Evolution of magnetic anisotropy in epitaxial Fe films by ferromagnetic resonance. J. Appl. Phys. Vol. 93, pp. 7622.

Zhang, Z.; Zhou, L.; Wigen, P.E.; Ounadjela, K. (1994 (a)). Angular dependence of ferromagnetic resonance in exchange coupled $\mathrm{Co} / \mathrm{Ru} / \mathrm{Co}$ trilayer structures, Phys. Rev. B. Vol. 50, pp. 6094. Zhang, Z.; Zhou, L.; Wigen, P.E.; Ounadjela, K. (1994 (b)). Using 
ferromagnetic resonance as a sensitive method to study the temperature dependence of interlayer exchange coupling. Phys. Rev. Lett. Vol. 73, pp. 336.

Zhang, B.; Cheng, J.; Gonga, X.; Dong, X.; Liu, X.; Ma, G.; Chang, J. (2008). Facile fabrication of multi-colors high fluorescent/superparamagnetic nanoparticles. J. Colloid Interf. Sci. Vol. 322, pp. 485.

Zhu, J.; Katine, J.A.; Rowlands, G.E.; Chen, Y.J.; Duan, Z.; Alzate, J.G.; Upadhyaya, P.; Langer, J.; Amiri, P.K.; Wang, K.L.; Krivorotov, I.N. (2012). Voltage-induced ferromagnetic resonance in magnetic tunnel junctions. ArXiv: 1205-2835: Cond-mat. Meshall. pp. 1. 